\title{
Proanthocyanidin synthesis in Theobroma cacao: genes encoding anthocyanidin synthase, anthocyanidin reductase, and leucoanthocyanidin reductase
}

Yi Liu ${ }^{1,4}$, Zi Shi', Siela Maximova ${ }^{2}$, Mark J Payne $^{3}$ and Mark J Guiltinan² ${ }^{2 *}$

\begin{abstract}
Background: The proanthocyanidins (PAs), a subgroup of flavonoids, accumulate to levels of approximately $10 \%$ total dry weight of cacao seeds. PAs have been associated with human health benefits and also play important roles in pest and disease defense throughout the plant.

Results: To dissect the genetic basis of PA biosynthetic pathway in cacao (Theobroma cacao), we have isolated three genes encoding key PA synthesis enzymes, anthocyanidin synthase (ANS), anthocyanidin reductase (ANR) and leucoanthocyanidin reductase (LAR). We measured the expression levels of TCANR, TCANS and TCLAR and PA content in cacao leaves, flowers, pod exocarp and seeds. In all tissues examined, all three genes were abundantly expressed and well correlated with PA accumulation levels, suggesting their active roles in PA synthesis. Overexpression of TCANR in an Arabidopsis ban mutant complemented the PA deficient phenotype in seeds and resulted in reduced anthocyanidin levels in hypocotyls. Overexpression of TcANS in tobacco resulted in increased content of both anthocyanidins and PAs in flower petals. Overexpression of TCANS in an Arabidopsis /dox mutant complemented its PA deficient phenotype in seeds. Recombinant TCLAR protein converted leucoanthocyanidin to catechin in vitro. Transgenic tobacco overexpressing TCLAR had decreased amounts of anthocyanidins and increased PAs. Overexpressing TCLAR in Arabidopsis /dox mutant also resulted in elevated synthesis of not only catechin but also epicatechin.
\end{abstract}

Conclusion: Our results confirm the in vivo function of cacao ANS and ANR predicted based on sequence homology to previously characterized enzymes from other species. In addition, our results provide a clear functional analysis of a LAR gene in vivo.

\section{Background}

Flavonoids are a diverse group of plant secondary metabolites with various biological functions that play important roles during plant development. They are involved in plant defenses against insects, pathogens and microbes, in absorption of free radicals and UV light, and in attraction of beneficial symbionts and pollinators [1-4]. Proanthocyanidins (PAs, also known as condensed tannins) are components of metabolites synthesized through the general flavonoid pathway. Their main

\footnotetext{
* Correspondence: mjg9@psu.edu

${ }^{2}$ Department of Plant Science, The Pennsylvania State University, 422 Life

Sciences Building, University Park, PA 16802, USA

Full list of author information is available at the end of the article
}

known function is to provide protection against microbial pathogens and invasions of insects and herbivores through multiple mechanisms [1]. PAs have metal chelating activity that results in severe limitation of bacterial growth; PAs can also associate with and irreversibly precipitate proteins, which is responsible for the astringent taste that repels herbivores. Furthermore, PAs can be oxidized to quinones which not only are powerful antibiotics themselves, but also can initiate cross-linking of cell walls to increase the strength of this physical barrier to pathogens [5-8]. In addition to these functional roles in plants, PAs, especially those from cacao, have recently been suggested to be beneficial to humans by improving cardiovascular health through activation of nitric oxide

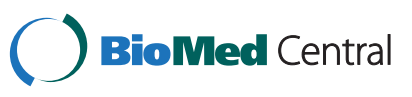

(c) 2013 Liu et al.; licensee BioMed Central Ltd. This is an open access article distributed under the terms of the Creative Commons Attribution License (http://creativecommons.org/licenses/by/2.0), which permits unrestricted use, distribution, and reproduction in any medium, provided the original work is properly cited. 
synthase, by providing cancer chemopreventative effects, and also through neuroprotective activities [9-11].

The biosynthetic pathways that lead to the production of flavan-3-ols $((+)$-catechin and (-)-epicatechin), the building blocks of PAs, have been well studied in the model plant species maize (Zea mays) and Arabidopsis as summarized in Figure 1 [12,13]. Biosynthesis of flavan-3-ols involves three principal enzymes: leucoanthocyanidin reductase (LAR), anthocyanidin synthase (ANS; also called leucoanthocyanidin dioxygenase, LDOX), and anthocyanidin reductase (ANR; in Arabidopsis, the product of BANYULS gene). The synthesis of PAs and anthocyanins share common steps leading to flavan3,4-diols (such as leucoanthocyanidin), which can be converted to catechin (2,3-trans-flavan-3-ol) by LAR [14] or to anthocyanidin by ANS $[15,16]$. Anthocyanidin then either serves as the substrate for the synthesis of epicatechin (2,3-cis-flavan-3-ol) by ANR [17], or can otherwise be converted to anthocyanin by glycosylation [18]. Both catechin and epicatechin act as the initiators for PA polymerization, with intermediates derived from leucoanthocyanidin, catechin or epicatechin added sequentially as extension units [1]. However, the details of the polymerization process are unclear and it is not known whether this is a spontaneous or an enzyme-catalyzed reaction. Recent work has identified two new enzymes downstream of flavan-3-ols that are involved in key steps of PA polymer biosynthesis, an epicatechin 3'-O-glucosyltransferase in Medicago truncatula [19] and MATE transporters from Arabidopsis and Medicago truncatula [20] that transport epicatechin 3'-O-glucoside to the vacuole in which it is likely that PA polymerization occurs [21].

ANS and ANR genes have been biochemically and genetically characterized in Arabidopsis [15,17,22,23], Medicago truncatula [17,24] and Vitis vinifera (grape) [25]. The Arabidopsis ans (ldox) mutant exhibits a deficiency in both anthocyanin accumulation in hypocotyls and PA deposition in seeds that results in a transparent testa phenotype [15]. Seeds of the Arabidopsis banyuls (anr) mutant exhibit a lack of PAs and a hyperaccumulation of anthocyanins, resulting in a dark red color reminiscent of the famous Banyuls wine produced in southern France [22]. Over-expression of $A N R$ genes from Medicago and grape in tobacco results in a loss of

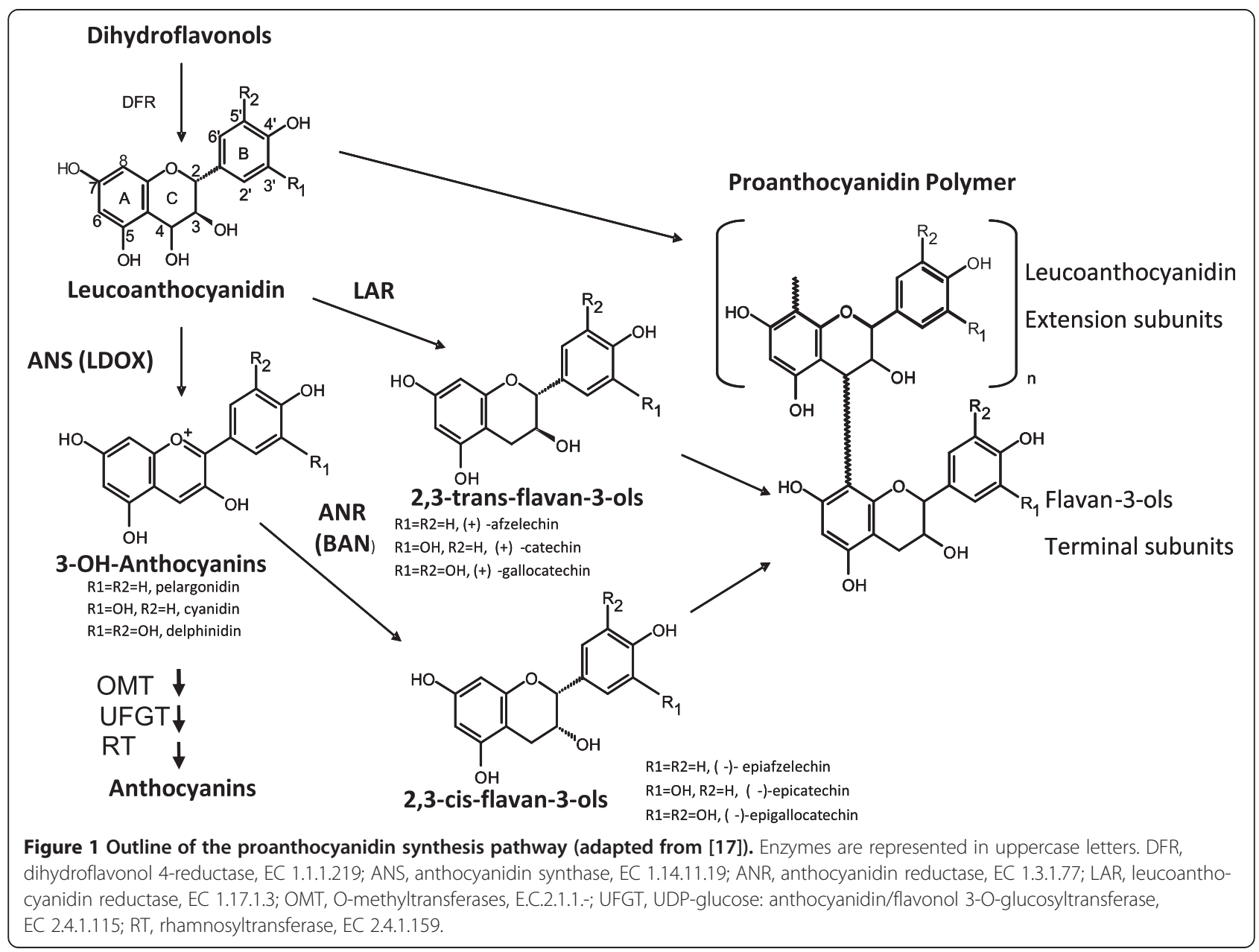


anthocyanin pigments in flower petals and elevated levels of PAs [17,25]. Antisense down-regulation of ANS in Medicago results in reduction of both anthocyanins in leaves and PAs in seeds [24].

$L A R$ genes have been isolated from various plant species including Desmodium uncinatum [14], Vitis vinifera [25], Lotus corniculatus [26] and Medicago truncatula [24] and their corresponding protein functions have been characterized by in vitro recombinant enzyme assays. However, the genetic evidence for $L A R$ function is rather indirect and less convincing than it is for $A N R$ and ANS, as discussed by Pang et al. [24]. It appears that the genomic sequence of Arabidopsis thaliana does not contain an intact $L A R$ orthologue, and correspondingly, catechin is not detected in Arabidopsis seed extracts $[12,14,15]$. LAR genes are expressed in other plant species that accumulate not only epicatechin but also catechin $[14,25,26]$. For example, grape and Lotus express both $L A R$ and $A N R$ genes and synthesize PAs consisting of both catechin and epicatechin [25-27]. In Medicago, although both $L A R$ and ANR are expressed, the PAs are composed almost entirely of epicatechin [24].

In Arabidopsis and Medicago, PA accumulation and gene expression is quantitatively and spatially limited to seed coats, making it remarkably difficult for biochemical analysis [12,24]. In contrast, Theobroma cacao (Tc) produces significant amounts of PAs in various tissues including leaves and beans; up to $12 \%$ of dry weight in leaves [28] and approximately $10 \%$ in mature beans [29]. Furthermore, large amounts of catechin and epicatechin monomers as well as their related polymers of different lengths have been detected in cocoa powder $[28,30]$. Considering the wide range of health benefits suggested for PAs and its significance for plant resistance, we targeted this pathway for molecular-genetic analysis.

This manuscript describes the isolation and expression of the TcANR, TcANS, and TcLAR genes encoding the key enzymes in proanthocyanidin biosynthesis. We measured PA content in different cacao tissues and performed functional characterization of the TcANR, TcANS, and TcLAR gene products through in vivo tests. The results presented here provide background and genetic tools that will be useful in the development of new cacao varieties with altered PA profiles.

\section{Results \\ Molecular cloning and sequence analysis of TcANR, TCANS and TCLAR genes}

The general pathway for the synthesis of proanthocyanidin and anthocyanins indicates the key enzymes ANR, ANS and LAR that carry out the biochemical steps at a critical metabolic branch point (Figure 1). To explore the genetic control of this important pathway in cacao, putative TcANR, TcANS and TcLAR cDNA sequences were identified in a collection of Theobroma cacao expressed sequence tags (ESTs) [31] by querying the cacao ESTtik database (http://esttik.cirad.fr/) with protein sequences of Arabidopsis BANYULS (NP_176365), Arabidopsis LDOX (Q96323) and Desmodium LAR (CAD79341). ESTs similar to each gene were assembled into contigs to determine consensus full-length open reading frames (ORF) by alignment with cDNAs of homologous genes from other species and predictions from the ORF Finder program (www.ncbi.nlm.nih.gov/ projects/gorf/). Full-length cDNAs of each gene were amplified by reverse transcription-PCR (RT-PCR) using RNA isolated from young leaves of cacao (Scavina 6). The TcANR cDNA (GU324348) contained a 1,008-bp open reading frame (ORF) encoding a protein of 336 amino acids that showed a $63 \%$ identity with the Arabidopsis BANYULS gene at the amino acid level. The TcANS cDNA (GU324350) contained an ORF of 1,062bp, which encodes a protein of 354 amino acids with $82 \%$ amino acid identity with the Arabidopsis $L D O X$ gene. The TcLAR cDNA (GU324352) contained a 1,083bp ORF encoding a protein of 361 amino acids with $61 \%$ amino acid identity with the Desmodium LAR protein.

To determine the genomic sequences of the putative cacao ANS, ANR and LAR genes, the cDNAs were used to screen a cacao BAC library by hybridization. A portion of each hybridizing BAC clone was sequenced using primers designed from the corresponding cDNAs. The genomic structure of each gene was established by alignment with its cDNA sequence (Figure 2). We also retrieved gene models of the ANS and ANR gene from Arabidopsis (AT4G22880 and AT1G61720) and LAR from Medicago and grape (BN000703 and NC_012007 c2622652-2619277) and compared them with the corresponding cacao genes.

The coding region of TcANR (GU324347) consisted of 6 exons and 5 introns distributed over 2,005-bp. The coding region of TcANS (GU324349) was shorter, having only 1,418-bp and consisting of 2 exons and 1 intron. The genomic organizations of these two cacao genes were nearly identical to the corresponding Arabidopsis genes, which had the same exon and intron numbers. Moreover, the lengths of the exons were very similar between Arabidopsis and cacao: for ANR, the numbers of nucleotides were precisely the same for the exons 2 , 3 , 4 , and 5 , although the lengths of introns were more variable.

Since $L A R$ does not have an orthologue in Arabidopsis, we compared the genomic organization of TcLAR with MtLAR and $V v L A R$ (Figure 2). The genomic organization of the coding region of TcLAR (GU324351) was similar to both MtLAR and $V v L A R$, consisting of 5 exons and 4 introns. As observed with the $A N R$ gene, the middle three exons (exon 2, 3 and 4) of $L A R$ from 


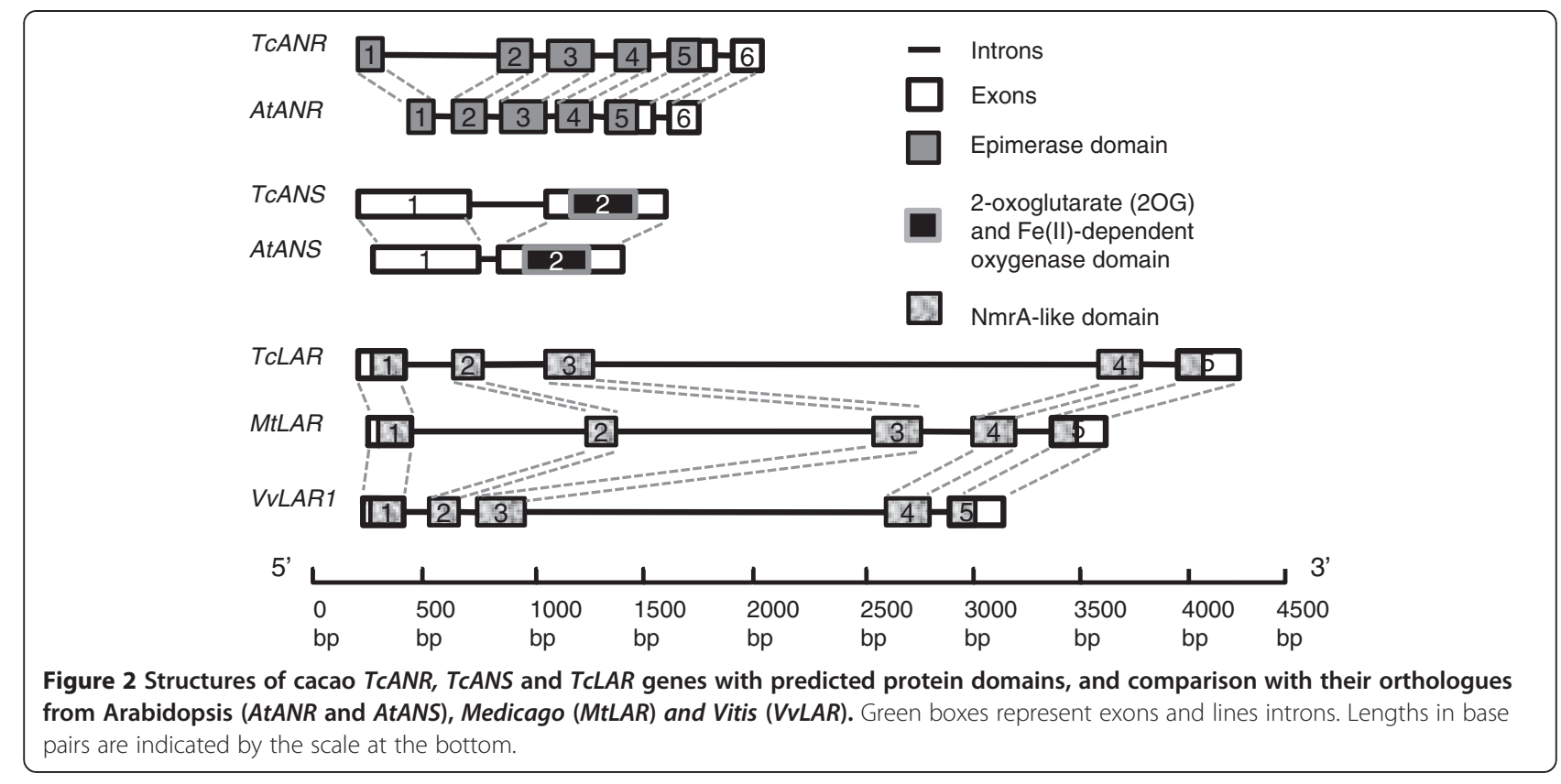

all three species had identical lengths. TcLAR exhibited an extremely long third intron of 2,338 bp; similarly, $V v L A R$ also featured a long third intron of 1,661 bp, while $M t L A R$ contained two long introns (intron 1 and intron 2) that are 812-bp and 1,178-bp respectively.

The analysis of the complete cacao genome sequence of the criollo variety was consistent with these results and allowed us to localize the position of each gene relative to the molecular-genetic map [32]. The ANR gene was located on chromosome 6 (Tc06_g018030, GenBank: GU324347.1) and was 99.75 identical to the cDNA described above. Similarly, a single ANS gene was located on chromosome 3 (Tc03_g026420, GenBank: GU324349.1) and shared 99.3\% identity to the cDNA described above. The $L A R$ gene was also located on chromosome 3 Tc03_g002450, GenBank: GU324351.1) and shares $98.5 \%$ identity to its corresponding cDNA. However, two more distantly related genes were identified in the cacao genome assembly that were not identified in the cacao EST collection. One gene (Tc05_p002410) encodes a protein that shares only $37 \%$ identity and $57 \%$ similarity with the TcANS protein and a second (Tc02_g034610) shares 63\% identity and 76\% similarity with the TcLAR protein. For this study, we focused our further work on the three genes of highest similarity to the well-characterized corresponding Arabidopsis and Desmodium genes.

LAR and ANR proteins belong to the reductaseepimerase-dehydrogenase (RED) superfamily, although their relationships are rather distant. ANS belongs to a different protein superfamily, the 2-oxoglutarate-dependent dioxygenase (2-ODD) superfamily, although it shares the same substrate with LAR (Figure 1). A phylogenetic tree was constructed using the neighbor-joining method with the sequences of functionally-tested proteins of LAR, ANR, ANS and DFR from various plant species. The tree construction also included all the IFR-like proteins from Arabidopsis that are most closely related to LAR proteins (Figure 3, see protein alignment in Additional file 1: Figure S1). The tree was noticeably bifurcated into two clades: all RED proteins (including ANR, LAR, DFR and IFR) constituting one clade, and all ANS proteins constituting the other. Within the RED superfamily clade, the IFR, LAR, ANR and DFR proteins are clearly divided into four distinct groups with IFR and LAR forming a subgroup that is distantly related to the subgroup formed by DFR and ANR groups. The cacao ANR, ANS and LAR proteins used in the current research all clustered within their own groups.

\section{Expression profiles of TCANR, TcANS and TcLAR genes}

To assess the involvement of TcANR, TcANS and TcLAR in PA biosynthesis in cacao, transcript levels were investigated in various tissues including leaves, flowers and pod tissues (Figure 4A, tissue definition and collection are described in Methods). Gene transcripts levels were assessed by semi-quantitative RT-PCR. TcActin was chosen as a reference to normalize gene expression because both cacao microarray analyses (Z. Shi and S. Maximova, unpublished data) and data from this study suggested a relatively constant spatial and temporal expression of this gene for all cacao tissues examined. PA synthesis genes, TcANR, TcANS and TcLAR were expressed in all tissues examined, with relatively higher expression levels in pod exocarp and seeds and lower expression in leaves and flowers. Moreover, their expression levels were relatively similar within each tissue 


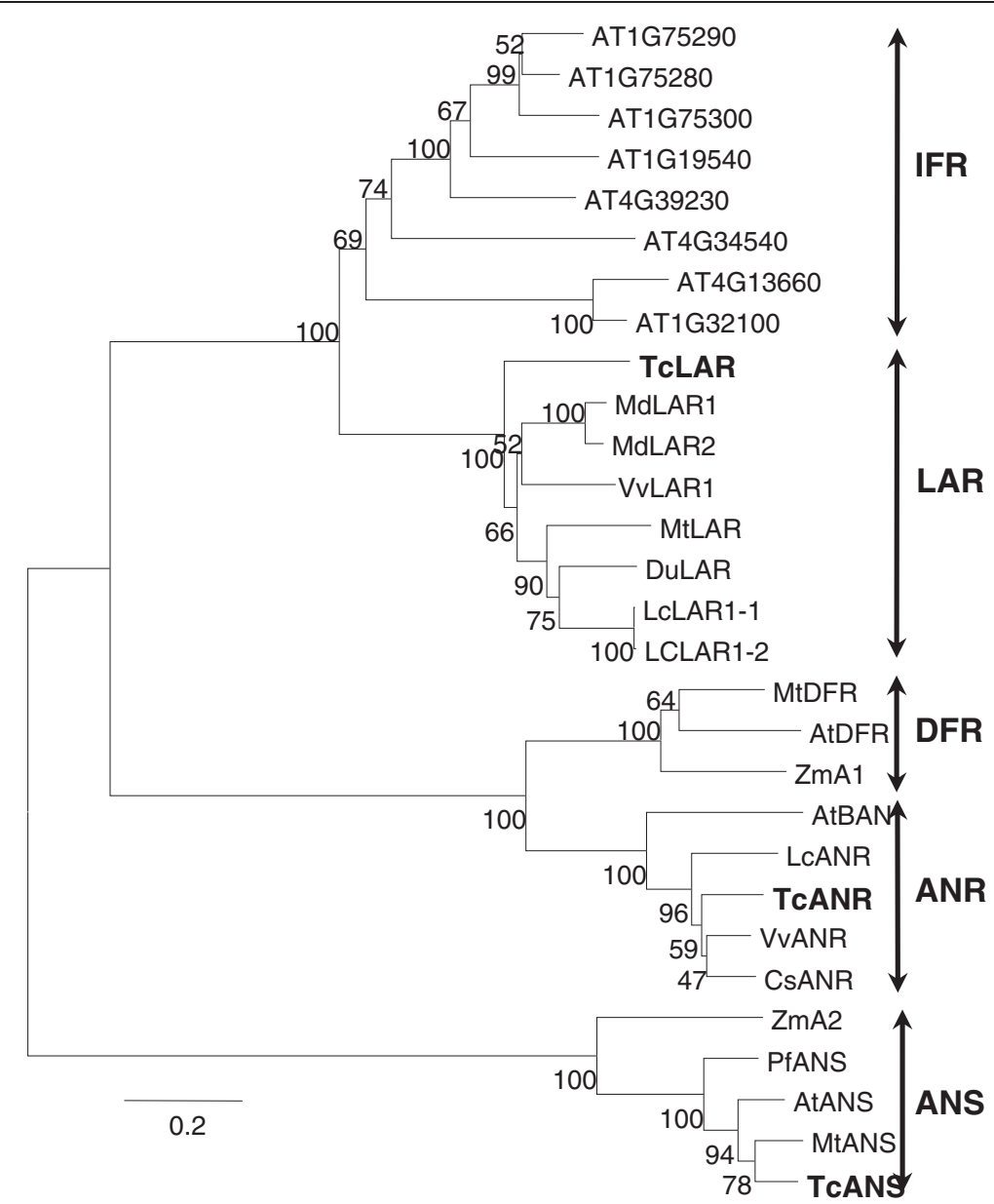

Figure 3 Phylogenetic analysis of the LAR, ANS and ANR proteins as well as related IFR and DFR proteins of the RED superfamily. The protein sequences were aligned as described in Material and Methods. The scale bar represents 0.2 substitutions per site. Numbers indicate bootstrap values. The tree includes LAR, ANR and ANS proteins from cacao, which are shown in bold font, as well as from other species whose enzymatic activities have been shown in previous publications. Also included in the tree are 8 IFR like proteins from Arabidopsis (labeled with their locus numbers). All the other proteins are labeled by the species they come from followed by their catalytic activities. The species represented and their GenBank accession numbers in the LAR group are Medicago (MtLAR, CAl56327), Vitis vinifera (VvLAR, CAI26309), Desmodium uncinatum (DuLAR, CAD79341), Malus domestica (MdLAR1, Q5D7Y1; MdLAR2, Q5D7Y2) and Lotus corniculatus (LCLAR1-1, ABC71324; LCLAR1-2, ABC71325); in the DFR group are Zea mays (ZmA1, CAA28734), Arabidopsis thaliana (AtDFR, NP_199094) and Medicago truncatula (MtDFR, AAR27014); in the ANR group are Arabidopsis thaliana (BAN, NP_176365), Medicago truncatula (MtANR, AAN77735), Vitis vinifera (VvANR, CAD91911), Camellia sinensis (CSANR, AAT68773) and Lotus corniculatus (LCANR, ABC71337); and in the ANS group are Perilla frutescens (PfANS, O04274), Arabidopsis thaliana (AtANS, Q96323), Zea mays (ZmA2, CAA39022) and Medicago truncatula (MtANS, ABU40983). The sequence alignment used to generate this figure can be found in "Additional file 1: Figure S1".

except seeds, in which the expression level of TcLAR was much lower than TcANR and TcANS.

\section{PA levels in various cacao tissues}

To determine the concentrations of PAs in different cacao tissues, samples were first extracted to obtain a soluble PA fraction. The residues left after soluble PA extraction were assayed using butanol- $\mathrm{HCl}$ (Yu-Guang [33]) to measure the amount of insoluble PAs represented as larger polymers. Because insoluble PA polymers will crystallize and bind to proteins and cell wall components, this interference may reduce the extraction efficiency of the insoluble PAs $[24,34]$. As a result, comparing the relative amount of these two fractions within the same tissue is difficult. However, the accumulation pattern of each fraction is comparable among different tissues. High levels of both soluble and insoluble PAs were detected in all tissues examined, ranging from approximately $40 \mathrm{mg} / \mathrm{g}$ DW in seeds to more than $170 \mathrm{mg} / \mathrm{g} \mathrm{DW}$ in exocarp for soluble PAs (Figure 4B) and ranging from approximately $1.2 \mathrm{mg} / \mathrm{g}$ DW in seeds to $8 \mathrm{mg} / \mathrm{g}$ DW in exocarps for insoluble PAs (Figure 4C). The accumulation of both soluble and insoluble PAs were in good correlation with the expression patterns of 


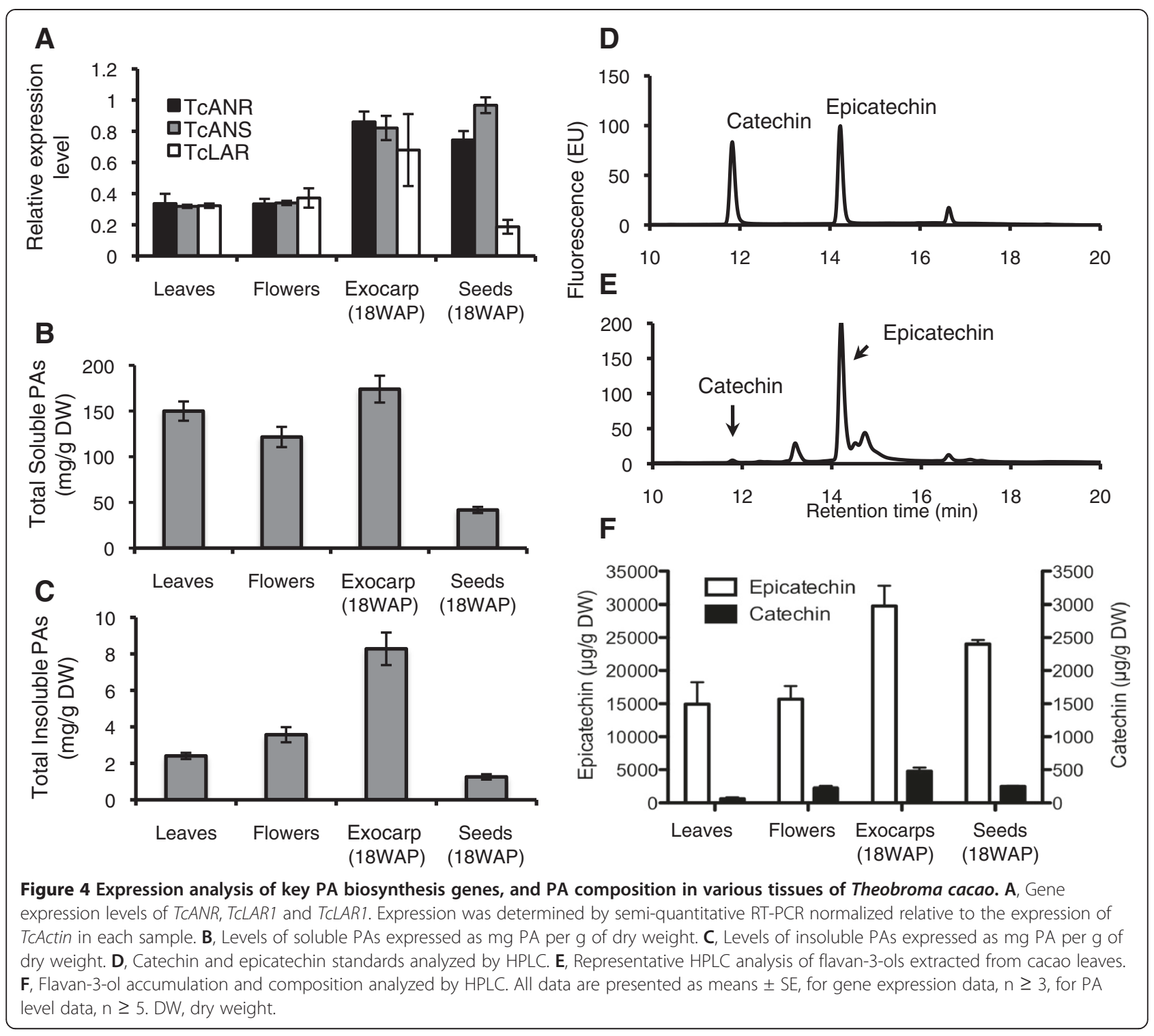

PA synthesis genes, both of which showed highest levels in fruit exocarp. However, both soluble and insoluble PA levels were much lower in seeds than in other tissues, which correlated with TCLAR but not TcANR and TcANS.

To determine the composition of monomer PAs in cacao, soluble PAs extracts prepared from all four tissues were further separated and quantified by HPLC. The data are presented for catechin and epicatechin concentrations as $\mu \mathrm{g}$ of catechin or epicatechin per g of dry tissue (Figure 4D-F). PAs consisted almost entirely of epicatechin with less than $2 \%$ catechin. In all tissues examined, the accumulation of both catechin and epicatechin were well correlated with the expression of TcLAR and TcANR. In exocarp, where both TcLAR and TcANR had the highest expression among all tissues tested, both catechin and epicatechin were also at maximal levels. In leaves and flowers, where TcANR expression was much lower, epicatechin was found at correspondingly lower levels. Likewise in seeds, which exhibited a much lower expression of TcLAR, catechin levels were also much lower.

\section{Functional analysis of the TcANR gene}

While the sequences and expression patterns of the candidate cacao PA biosynthesis genes were consistent with their candidate functions relative to enzymes from other species, we conducted in vivo functional analysis of each gene in transgenic plants to gain direct evidence for their functions. To investigate the in vivo function of TcANR, a genetic complementation experiment was performed by transferring the TcANR coding sequence under the control of an enhanced expression promoter $(\mathrm{E} 12 \Omega$, a modified CaMV35S promoter having the 
omega arrangement derived from tobacco mosaic virus) into the Arabidopsis banyuls (ban) mutant, which is defective in the gene encoding ANR [22]. ban mutant seeds are pale yellow due to a lack of PAs, and there is more purple anthocyanin pigment deposited in hypocotyls compared to Arabidopsis (ecotype Columbia) control plants due to increased metabolic flux into this branch of the pathway. From eight independent transgenic TcANR-overexpressing lines tested, five lines showed white hypocotyls, and 3 lines showed reduced pigmentation in hypocotyls as compared to banyuls mutants. The lines with white hypocotyls also produced seeds exhibiting the wild-type phenotype that stained blue with DMACA reagent, suggesting the deposition of PAs in the seed coat (Figure 5A). After PA extraction and quantification, all lines showed significantly increased levels of PAs (Figure 5C). RT-PCR analysis confirmed expression of TcANR, and the expression levels positively correlated with PA accumulation (Figure $5 \mathrm{~B}$ ).

\section{Functional analysis of TcANS gene}

To investigate the in vivo function of TcANS, two model plants, tobacco and Arabidopsis were utilized. We used an Arabidopsis ans (ldox) mutant to perform tests of transgenic complementation. We used tobacco as a model system for metabolic flux analysis of the equilibrium between PA and anthocyanin synthesis. Introduction of functional ANS genes into tobacco can result in flower petal color changes reflecting alterations in ANS activity.

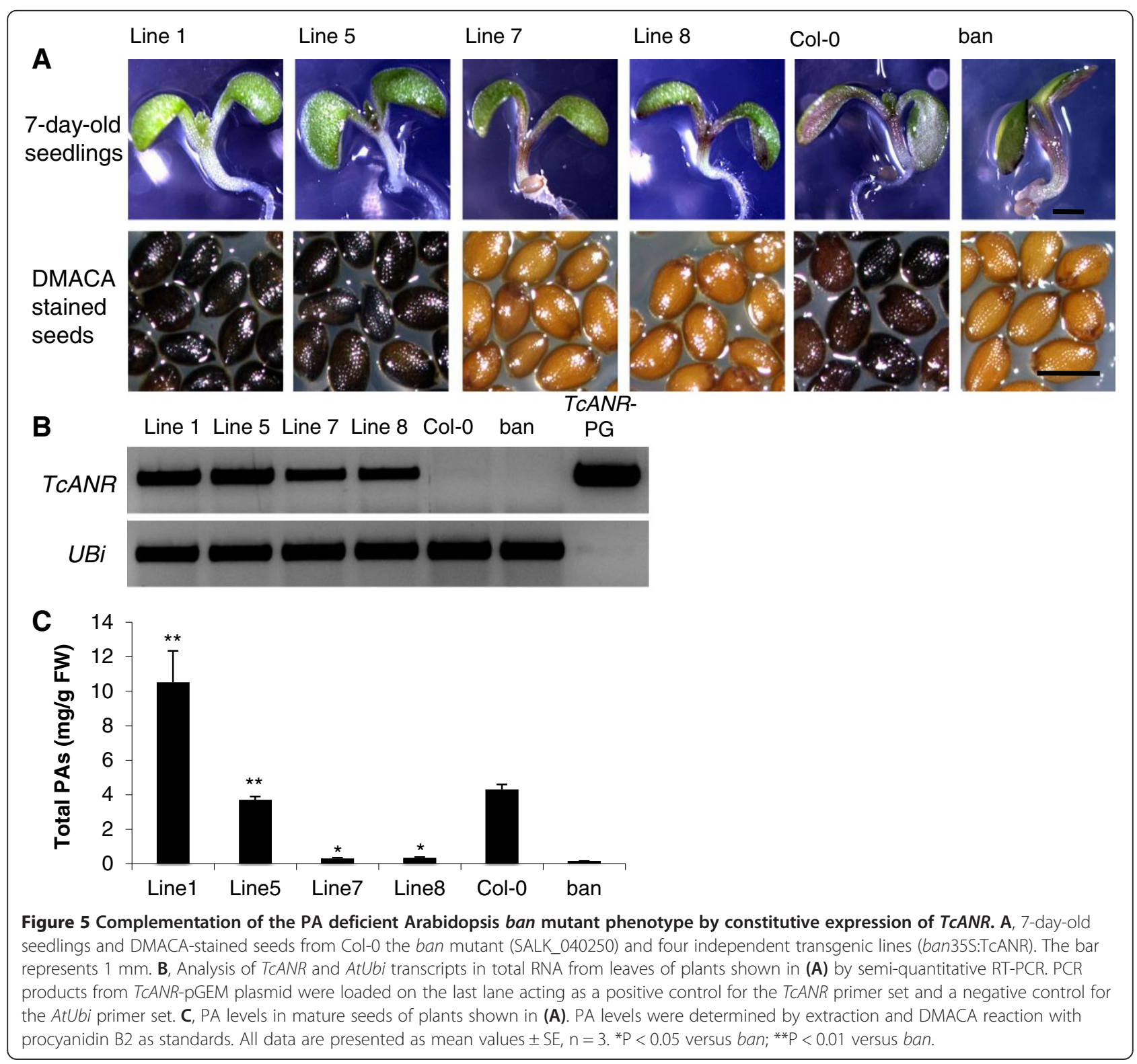


ANS is involved in both PA synthesis and anthocyanin synthesis, as cyanidin, an anthocyanin precursor, can also be reduced to epicatechin by ANR (Figure 1). This was demonstrated in a recent study describing that down regulation of MtANS in Medicago resulted in decreased levels of both PAs and anthocyanins [24]. Based on these results, we reasoned that if the putative cacao ANS protein is truly a functional ANS, over-expression of TcANS in tobacco should result in increased accumulation of both anthocyanins and PAs. The ORF of TcANS, driven by the E12 $\Omega$ promoter, was introduced into wild-type tobacco (cv. Samsun) for constitutive ectopic expression. Twelve independent hygromycinresistant lines were generated, of which nine showed a visible increase in pink color intensity in flower petals. The two lines displaying the greatest increase in petal color were chosen for further analysis. RT-PCR analysis confirmed high TcANS transcript levels in these two tobacco transgenic lines, which positively correlated with the color of the petals (Figure 6A and B). Amplification of the tobacco ribosomal RNA gene NtrRNA, which served as an internal control, showed a relatively similar expression level in both wild-type control and transgenic tobacco plants. As predicted, anthocyanin levels increased in the two transgenic lines (Figure 6C).

The levels of PAs in the petals of transgenic lines, quantified by DMACA assays, were also significantly higher as compared to untransformed Samsun plants (Figure 6D). On average, a two-fold increase of PA was observed in the two lines compared to wild type.

The 35S-TcANS transgene was also introduced into the Arabidopsis ans (ldox) T-DNA mutant, which produces hypocotyls that appear white to light green due to lack of anthocyanins, and seeds that appear light yellow due to lack of PAs. Eighteen independent hygromycin resistant transgenic T1 seedlings were selected. From these, 2 lines developed wild-type purple colored hypocotyls (Additional file 1: Figure S2) and produced wildtype brown-colored seeds that stained blue after reacting with the DMACA reagent. The color suggested deposition of PAs in the seed coat, however the color intensity was lower than in wild type. RT-PCR using RNA extracted from T2 seedlings confirmed expression of TcANS genes (data not shown).

\section{Functional analysis of the TcLAR gene}

Because the $L A R$ gene is not present in Arabidopsis [15], we cannot obtain direct evidence of the in vivo function of TcLAR through genetic complementation analysis in Arabidopsis. Therefore, to functionally characterize $T c L A R$, we used tobacco as a model system. As overexpression of the $A N R$ gene can divert the metabolic flow from anthocyanin synthesis to PA synthesis [17], we predicted that over-expression of the cacao $L A R$ gene in tobacco would result in a decrease in anthocyanin pigment and an increase in PA accumulation in flower petals. Transgenic tobacco plants were generated that constitutively expressed the ORF of TcLAR under the control of the E12 $\Omega$ promoter. Twenty-two independent transgenic lines that are resistant to hygromycin were generated. Nine of these exhibited a decrease in intensity of the visible pink color of petals. Two lines (Lines LAR7C1 and LAR9A2) exhibited virtually white petals (Figure 6E). RT-PCR showed that both lines expressed high levels of TcLAR transcripts (Figure 6F). Quantification of soluble PA and anthocyanin levels indicated that anthocyanin levels in these two lines were about half those of WT controls, and that total PAs accumulated to an approximately 5-fold higher level than in controls (Figure 6G and $\mathrm{H}$ ).

Moreover, the levels of PAs in transgenic tobacco petals were inversely proportional to the concentrations of anthocyanin, indicating diversion of metabolic flow from anthocyanin to PA synthesis. Insoluble PA levels were also estimated, but the levels were insignificant (data not shown). To confirm the increased accumulation of catechin, as it is the predicted product of TcLAR, we separated and quantified the PA extracts using HPLC. The HPLC analysis showed that there is indeed an approximately 2-fold increase of catechin levels in both transgenic lines. Surprisingly, there is also a significant increase of epicatechin levels (Figure 6I).

We also took advantage of the Arabidopsis ans (ldox) T-DNA mutant to examine $L A R$ function. Because Arabidopsis lacks an $L A R$ gene $[12,14,15]$ and the ldox mutant is deficient in cyanidin (the substrate for $A N R$ ), the ldox mutant exhibits a significant decrease of epicatechin and PA synthesis. We reasoned that since the ldox mutant accumulates leucoanthocyanidin which can provide the substrate for a heterologous LAR protein, the ldox mutant was potentially a good in vivo model to analyze LAR function. We predicted that overexpression of TcLAR should result in the synthesis of catechin in developing siliques of the ldox mutant Arabidopsis, even if PAs were not produced due to lack of epicatechin synthesis. HPLC separation and quantification of PA extracts from Arabidopsis transgenic lines over-expressing TcLAR gene in the ldox mutant background revealed not only a significant increase of catechin, but also surprisingly, a modest increase of epicatechin (Figure 7). Quantification of total PAs extracted from mature seeds also revealed significant PAs increases in all transgenic lines compared to the ldox mutant (Additional file 1: Figure S4).

These unexpected results prompted us to check the possibility that TcLAR may carry a dual enzymatic activity, capable of converting leucoanthocyanidin to both catechin and epicatechin. Thus, recombinant TcLAR 


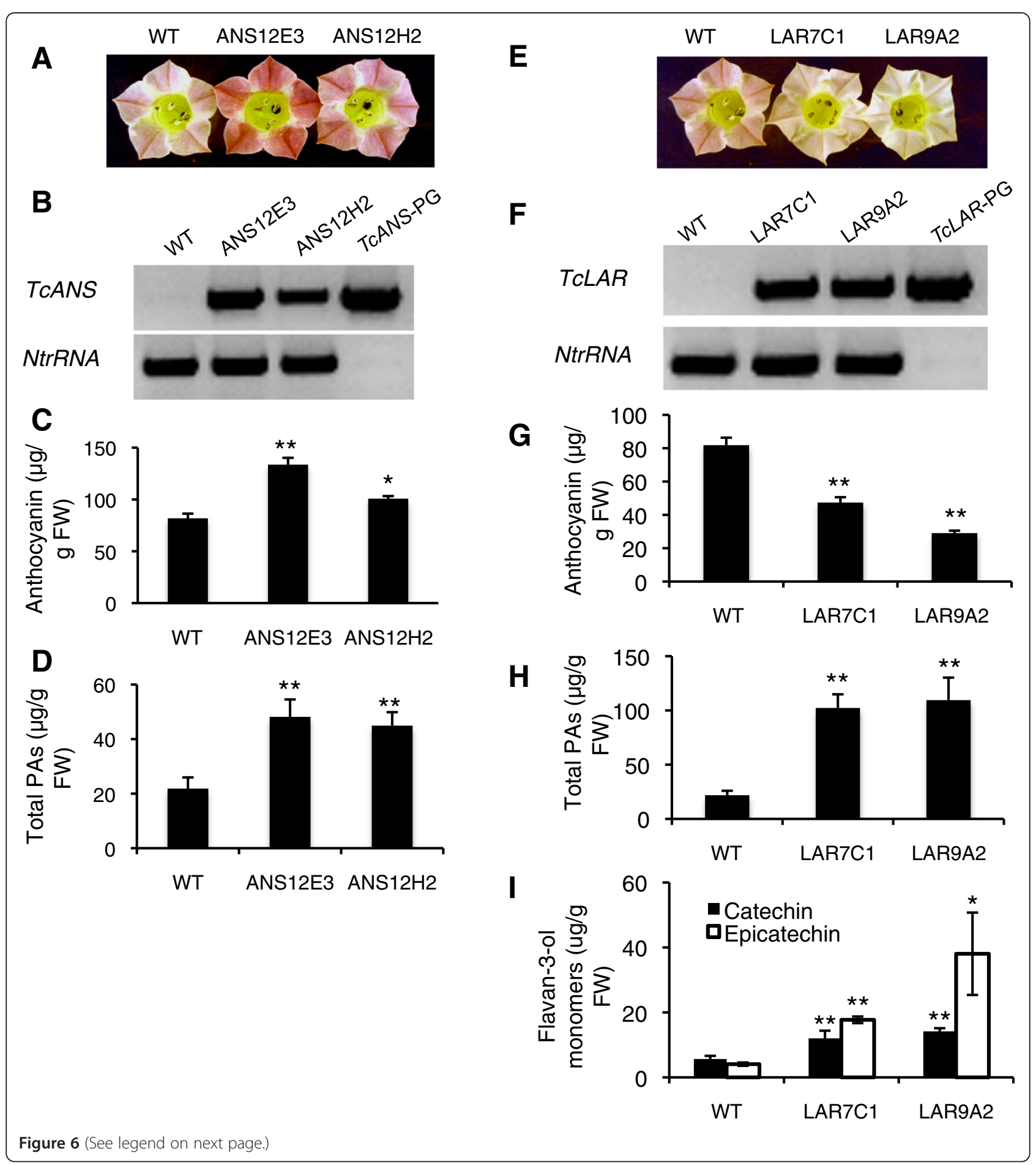


(See figure on previous page.)

Figure 6 Characterization of transgenic tobacco flowers constitutively expressing TCANS or TCLAR. A, Pigmentation of flower petals from wild type (WT) and two independent lines of TCANS transgenic (ANS12E3, ANS12H2) tobacco plants. B, Analysis of TCANS and NtrRNA transcripts in total RNA from leaves of plants shown in (A) by RT-PCR. PCR products from TCANS-pGEM plasmid were loaded on the last lane acting as a positive control for TCANS primer set and a negative control for the NtrRNA primer set. $\mathbf{C}$, Anthocyanin levels in flower petals of plants shown in (A). D, Total soluble PA levels in flower petals of plants shown in (A). E, Flowers from wild type (WT) and two independent lines of TCLAR transgenic (LAR7C1, LAR9A2) tobacco plants. F, Analysis of TCLAR and NtrRNA transcripts in total RNA from young leaves of plants shown in (E) by RT-PCR. PCR products from the TCLAR-PGEM plasmid alone were loaded on the last lanes to act as a positive control for the TCLAR primer set and a negative control for the NtrRNA primer set. G, Anthocyanin levels in flower petals of WT and transgenic plants. $\mathbf{H}$, Total soluble PAs levels in flower petals of WT and transgenic plants. I. Flavan-3-ol accumulation and composition in flowers of wild type (Samsun, ss) tobacco plants and two independent lines of 35S:TCLAR transgenic (LAR7C1, LAR9A2) tobacco plants. Anthocyanin levels were determined by extraction and UV absorption with cyanidin 3-glucoside as standards. Total soluble PA levels are determined by extraction and DMACA reaction with procyanidin B2 as standards. Flavan-3-ol levels were determined by extraction, HPLC separation, and quantification. All data are presented as mean values $\pm \geq$ SE,7. ${ }^{*} \mathrm{Pn}<0.05$ versus $\mathrm{WT}$; ${ }^{*} \mathrm{P}<0.01$ versus $\mathrm{WT}$.

protein was expressed in E. coli, purified and assayed using ${ }^{3} \mathrm{H}$-labeled leucocyanidin as substrate in the presence of $\mathrm{NADPH}$, followed by analysis of products by HPLC-UV and radioactivity detection. The negative control reaction (boiled protein) showed no product formation (Figure 8A and $\mathrm{C}$ ), whereas a peak with the same retention time and $\mathrm{UV}$-spectrum as that of the pure (-)-catechin standard (Figure 8E and F) was detected when TCLAR protein was incubated with ${ }^{3} \mathrm{H}$-labeled leucocyanidin (Figure 8B and D). However, no epicatechin product was detected (the retention time of 26-27 min), suggesting that TcLAR does not exhibit duel functionality in this in vitro assay.

\section{Discussion}

The distribution of cacao PA accumulation correlates with expression of the PA biosynthesis genes

The localization of PAs in plant tissues has been well studied in the model plants Arabidopsis, the legume Medicago truncatula, and in the fruit species grape (Vitis vinifera). In Arabidopsis, the expression of the AtANR (BANYULS) gene and PA accumulation is limited to the seed coat [22,35]. Similarly in Medicago the major localization of PAs is also in the seed coat with very small amounts present in flowers, leaves, roots and stems [24]. Although Theobroma cacao and Arabidopsis are phylogenetically closely related, the PA accumulation profiles are quite different. Our results demonstrated that in cacao leaves, flowers and fruits, PAs accumulate at high levels and this is correlated with expression of genes encoding the key enzymes involved in PA synthesis. This is similar to Vitis, in which both PA accumulation and genes involved in PA synthesis are found in leaves, fruit skins and seeds [25].

In cacao, the expression of $A N S, A N R$, and $L A R$ were co-regulated and their expression correlated well with PA accumulation in most of the tissues, suggesting significant roles in PA synthesis for both ANR and LAR. The only exception is in seeds, in which the expression level of TcANS is higher than TcANR and TcLAR is much lower. The high expression level of TcANS may contribute to anthocyanin synthesis, because at the time when seed tissues were harvested (18 WAP), anthocyanins are actively synthesized [36-38]. The PA level in seeds is also much lower than in other tissues, well correlated with the TcLAR but not TcANR. The LAR enzyme seems to be a rate-limiting factor of the PA synthesis. To further explore the individual contributions of these proteins to catechin and epicatechin synthesis, flavan-3-ol monomer composition was examined by HPLC analysis. Cacao flavan-3-ol monomers were composed almost entirely of epicatechin units with catechin units comprising less than $2 \%$ in all tissues examined. This result is consistent with aprevious study of PA synthesis in cacao beans [30], in which PA extension units were reported to be composed exclusively of epicatechin, and terminal units were mostly epicatechin with about $1 \%$ catechin. Similarly, Medicago PAs consist almost entirely of epicatechin units [24]. In grape, both ANR (encoded by $V v A N R$ ) and LARs (encoded by $V v L A R 1$ and $V v L A R 2)$ contribute to PA synthesis in the fruits, in which PAs consist of both catechin and epicatechin. However, in grape leaves, although catechin is still present, $L A R$ genes are expressed at very low levels.

\section{Functional characterization of cacao PA biosynthetic genes ANS, ANR and LAR}

In vivo genetic analysis of TcANS and TcANR verified their roles in PA biosynthesis. Over-expression of TcANS in tobacco resulted in elevated levels of both anthocyanins and PAs in flower petals. It also restored anthocyanin synthesis in hypocotyls as well as PA accumulation in seeds of the Arabidopsis ans (ldox) mutant. Similarly, over-expression of TcANR in the Arabidopsis banyuls (anr) mutant restored PA synthesis in seeds. Moreover, ectopic over-expression of TcANR in hypocotyls diverted the anthocyanin synthesis branch and resulted in decreased anthocyanin pigments related to gene expression levels. The catalytic activities of LAR proteins from grapevine, Medicago and Desmodium have been verified 


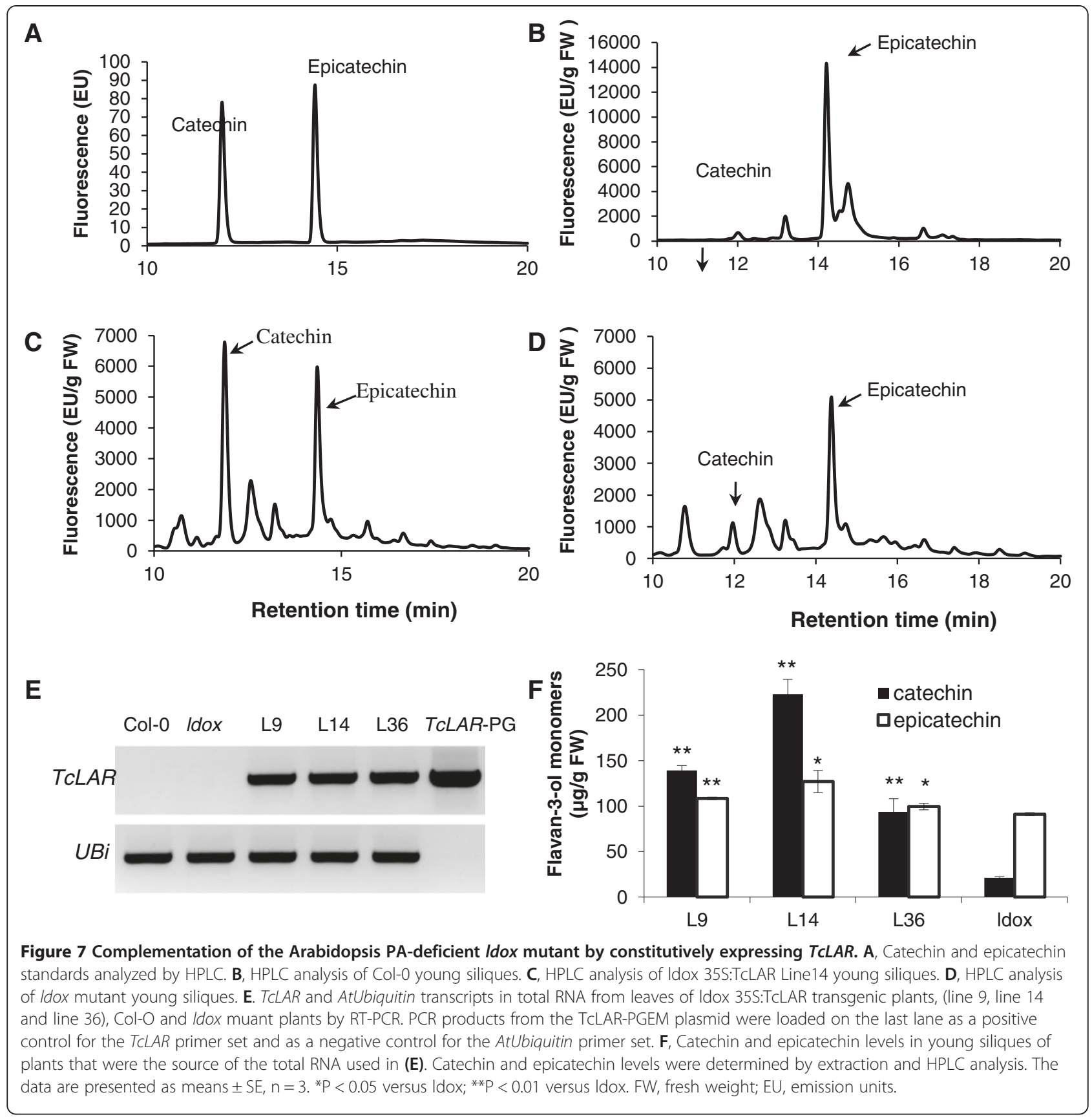

by in vitro assays of recombinant proteins [14,24,25]. Lotus LAR showed weak activity in a recombinant DFR protein coupled assay [26]. However, none of the enzyme assays was able to recover sufficient flavan-3-ol products for stereo chemical analysis to allow specific determination of catechin and epicatechin. The in vivo activities of Medicago and Desmodium LAR proteins was also tested in tobacco and white clover [14,24]. In the former study [14], the authors found that transgenic plants expressing the DuLAR protein showed slightly elevated LAR enzymatic activity compared to extracts from untransformed plants, but they did not demonstrate changes in metabolite synthesis. In a more recent study of $M t L A R$ overexpresing transgenic tobacco plants, there were no detectable changes in anthocyanin and PA levels [24]. The results presented in this work shows that a cacao TcLAR gene is co-regulated with TcANR in all tissues examined, providing a simple model to investigate LAR enzyme functions. In our results, TcLAR showed clear in vivo activities in both transgenic tobacco and Arabidopsis plants by converting metabolic flow from anthocyanin synthesis to PA synthesis. This 


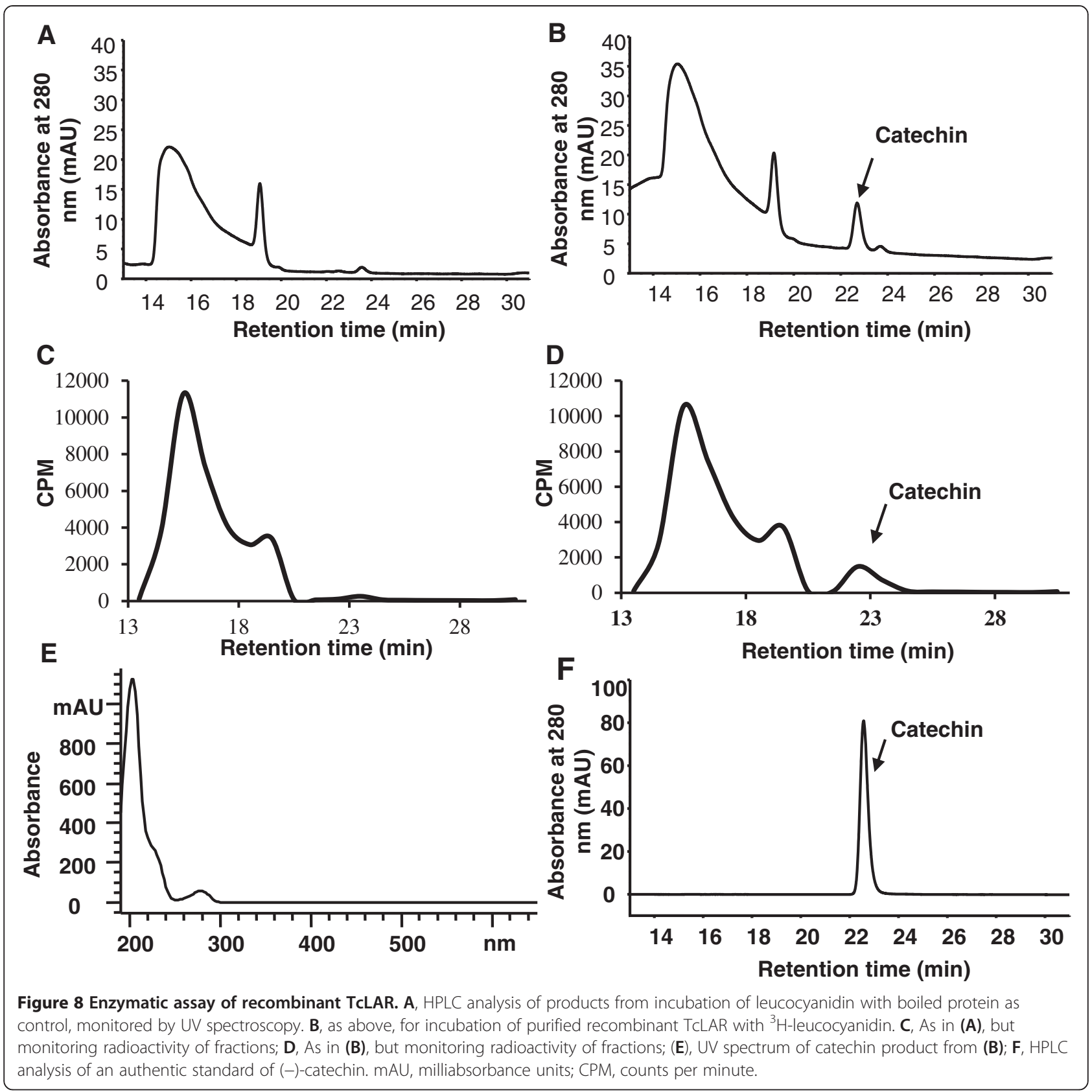

data provides a direct genetic evidence for a clear role of $L A R$ in PA biosynthesis. The metabolic flow divergence resulting from TcLAR over-expression could be due to perturbations of a hypothesized metabolic channeling mechanism that suggests that multiple enzymes in each subsequent step of the synthesis pathway interact as a means to increase the efficiency and throughput of the pathway [39].

To our surprise, ectopic expression of TcLAR resulted in elevated levels of both catechin and epicatechin units in tobacco flowers (Figure 6H) in contrast to the prediction that only catechin should be formed. Based on the sequence similarity between LAR and ANR, it is possible that LAR could function redundantly to ANR and convert cyanidin to epicatechin. Alternatively, LAR may perform as a dual functional enzyme and convert leucoanthocyanidin to both catechin and epicatechin. To test the possibility that LAR performs the same activity as ANR and uses cyanidin as a substrate to form epicatechin, we took advantage of the Arabidopsis ldox (ans) mutant. Since Arabidopsis does not have an $L A R$ gene and synthesizes only epicatechin through ANS and ANR pathway, the $l d o x$ mutants have a significantly reduced PA (epicatechin) level due to lack of a supply of 
cyanidin, the epicatechin precursor. Thus, the ldox mutant provides a system that has leucoanthocyanidin but not cyanidin. Over-expression of TcLAR in the ldox mutant resulted in synthesis of catechin confirming its predicted enzymatic function. However, HPLC quantification showed that there was only a slight elevation of epicatechin levels compared to the significant elevation of catechin, suggesting that LAR was less likely to perform dual function and convert leucoanthocyanidin to epicatechin.

When recombinant TCLAR was assayed in vitro, while we observed the production of catechin from 2,3-trans3,4-cis-leucoanthocyanidin, we did not detect even trace amounts of epicatechin. The failure to detect epicatechin in assays with the cacao LAR enzyme suggests that it functions solely in converting leucoanthocyanidin to catechin. However, it is possible that LAR enzyme can synthesize epicatechin from 2,3-cis-3,4-trans-leucocyanidin as suggested by [14]; but we could not test this possibility in the recombinant TcLAR enzyme assay because 2,3-cis-3,4-trans-leucocyanidin is not commercially available. The increase in epicatechin in TcLAR overexpressing Arabidopsis and tobacco plants could be due to the possible existence of a gene(s) encoding a catechin-epicatechin epimerase. One possible candidate gene that could be involved is ANR. Vitis vinifera ANR protein expressed and purified from E.coli showed epimerase activities in vitro and converted cyanidin to a mixture of catechin and epicatechin [40]. Similarly, two recombinant ANR proteins cloned from Camellia sinensis also showed epimerase activities in vitro [41]. However, we regard this as a remote possibility because Arabidopsis itself does not synthesize catechin and thus it would be surprising if a catechin-epicatechin-specific epimerase were expressed in the absence of its substrate. Nevertheless, we cannot exclude the possible existence of such an epimerase that may also serve other functions important in plant development. A second alternative hypothesis to explain epicatechin formation would be racemization of catechins by polymerization to proanthocyanidins followed by nonstereo-specific depolymerization. An earlier report lends some support to this idea through the analysis of transgenic apple lines in which the MdANS gene was silenced. The transgenic lines showed a drastic reduction in anthocyanins together with significant increase of both catechin and epicatechin, concomitant with an increase of catechin derived PA polymers and a decrease in epicatechin derived polymers [42]. Based on these observations, the authors of this study suggested that the increased epicatechins were derived from non-stereospecific depolymerization of proanthocyanidins.

A third possible explanation for our results is the hypothesis that increased production of catechin in the transgenic plants alters the metabolic equilibrium in such a way as to increase flux through the epicatechin branch as a feedback mechanism to balance the relative amounts of catechin and epicatechin. It is known that DuLAR activity can be inhibited by catechin with an IC50 as low as $12 \mu \mathrm{M}$ [14]. Feedback and feedforward mechanisms also exist at a transcriptional level in the flavonoid pathway. For example, a bean chalcone synthase (CHS) promoter can be stimulated by low concentrations of trans-cinnamic acid (CA), the first intermediate of the phenylpropanoid pathway while its activity can be repressed by high concentrations of CA [43]. On the other hand, high concentrations of trans-p-coumaric acid, the second intermediate of the phenylpropanoid pathway, can still stimulate the CHS promoter [43]. It seem likely that a complex web of homeostatic mechanisms function to control the flux through this entire pathway and elucidation of these mechanisms remains a major objective of this field for the future.

\section{Conclusions}

We successfully isolated three genes encoding key PA synthesis enzymes in Theobroma cacao, TcANS, TcANR, and TcLAR. In vivo genetic analysis of TcANS and TcANR in tobacco and complementation in Arabidopsis verified their roles in PA biosynthesis. In vitro enzyme assays of TcLAR recombinant protein verified its predicted function. Moreover, in vivo overexpressing TcLAR in tobacco and Arabidopsis ldox mutants successfully diverted metabolic flow to PA syntheis, which provide direct evidence for a clear role of the TcLAR gene in PA biosynthesis. Our results provide new knowledge and genetic tools for development of cacao varieties with novel PA profiles through conventional breeding or genetic approaches.

\section{Methods}

\section{Plant material}

Two Theobroma cacao varieties: Scavina 6 and Amelonado were used for this study. Cacao plants were grown in greenhouse as previously described [44]. Leaf and flower tissues were collected from Scavina 6 plants. For leave tissues, young red stage leaves were collected. The definition of leaves stages were previously described [45]. Cacao pods were obtained by hand pollinating Amelonado (a self-compatible variety), in order to reduce the effect of genetic variation on seed traits. Pods harvested 18 weeks after pollination were dissected into exocarp (outer fruit tissue) and seeds for separate analysis. Exocarp samples represent the outer 1-3 mm layer of the fruit obtained using a fruit peeler. All samples were frozen in liquid nitrogen upon collection and stored at $-80^{\circ} \mathrm{C}$ until extraction.

Transgenic and wild-type tobacco plants (Nicotiana tabacum var. Samsun provided by Wayne Curtis, Department of Chemical Engineering, The Pennsylvania 
State University) were grown in a greenhouse under the same condition as cacao plants. Arabidopsis plants (Arabidopsis thaliana) were grown in soil at $22^{\circ} \mathrm{C}, 50 \%$ humidity and a $16 \mathrm{~h} / 8 \mathrm{~h}$ light/dark photoperiod in a growth chamber (Conviron, Pembina, ND, USA). Plants grown aseptically were plated on MS medium [46] with $2 \%(\mathrm{w} / \mathrm{v})$ sucrose solidified with $0.6 \%(\mathrm{w} / \mathrm{v})$ agar. Arabidopsis ecotype Columbia ( $\mathrm{Col}-0)$ plants were used as the wild type. T-DNA insertion mutants ban (SALK_040250) and ldox (SALK_028793) were obtained from The Arabidopsis Biological Resource Center (Columbus, OH, USA).

\section{Nucleic acid purification and CDNA synthesis}

Total RNA from leaves of Theobroma cacao (Scavina 6) was isolated using a modified cetyl trimethyl ammonium bromide (CTAB) extraction method as previously described [47] with the following modifications. RNA isolated from the CTAB extraction $\mathrm{LiCl}$ precipitation was further purified and concentrated using RNeasy columns (Qiagen, Valencia, CA, USA), but the phenol/chloroform extraction and sodium acetate/ehanol precipitation step was omitted. The quality of RNA was verified by observing absorbance ratios of A260/A280 (1.8 to 2.0) and A260/A230 (1.8 to 2.2) and by separating 200 ng RNA samples on $0.8 \%$ agarose gels to examine intact ribosomal bands. First strand cDNA was synthesized using the SMART RACE cDNA amplification kit (Clontech, Mountain View, CA, USA).

\section{Isolation of CDNA and genomic clones from Theobroma cacao}

The putative expressed sequence tag (EST) sequences of cacao anthocyanidin reductase (TcANR), anthocyanidin synthase (TcANS) and leucoanthocyanidin reductase (TcLAR) genes were obtained by searching the Theobroma cacao EST database (http://esttik.cirad.fr/) [31] using the tBLASTn program [48]. The query sequences used were the protein sequences of BANYULS and LDOX from Arabidopsis thaliana and DuLAR from Desmodium uncinatum respectively (Accession numbers: NP_176365, Q96323 and CAD79341). Based on the sequences of the EST contigs from the ESTtik database (EST Treatment and Investigation Kit; http://esttik.cirad. fr), PCR primers were designed to amplify the entire coding sequences of each gene: ANR_F (5'-AGCCATGGCCAGCCAGACCGTAGG-3') and ANR_R (5'-GCGGCCGCTCA CTTGAGCAGCCCCTTAGC-3'), ANS_F (5'-CCATGGT GACTTCAATGGCCCCCAG-3') and ANS_R (5'-GCG GCCGCCTCAATTAGACAGGCCATC-3') and LAR_F (CCATGGATATGAAATCAACAAACATGAATGGTTC) and LAR_R (GCGGCCGCTCATGTGCATATCGCAGTG). $\mathrm{NcoI}$ sites were added to the $5^{\prime}$ end of each start codon and NotI sites were added to the 3' end of each stop codon to facilitate the subsequent cloning into binary T-DNA vectors. The coding sequences were amplified from cacao cDNA prepared from young leaves (genotype Scavina 6) with the Advantage cDNA PCR Kit (Clontech, Mountain View, CA, USA) using these primers. PCR reactions were carried out in a total volume of $20 \mu \mathrm{L}$ at $94^{\circ} \mathrm{C}$ for $5 \mathrm{~min}$; 5 cycles of $94^{\circ} \mathrm{C}$ for $30 \mathrm{sec}, 55^{\circ} \mathrm{C}$ for $30 \mathrm{sec}$, and $72^{\circ} \mathrm{C}$ for $1 \mathrm{~min}$; then another $23 \mathrm{cycles}$ of $94^{\circ} \mathrm{C}$ for $30 \mathrm{sec}, 60^{\circ} \mathrm{C}$ for $30 \mathrm{sec}$, and $72^{\circ} \mathrm{C}$ for $1 \mathrm{~min}$; followed by a final extension at $72^{\circ} \mathrm{C}$ for $5 \mathrm{~min}$. PCR products were gel purified and cloned into the pGEM-T easy vector (Promega, Madison, WI, USA). The correct open reading frames (ORFs) of each of the resulting constructs (pGEMT-TcANR, pGEMT-TcANS and pGEMT-TcLAR) were confirmed by DNA sequencing.

The DNA sequences of the TcANR, TcANS and TcLAR genes were obtained by isolation and sequencing of genomic clones. Briefly, 2 high-density filters were arrayed with 18,432 colonies of Theobroma cacao (genotype LCT-EEN 37) bacterial artificial chromosome (BAC) clones on each (library and filters constructed by The Clemson University Genomics Institute (CUGI, https:// www.genome.clemson.edu/). The filters were hybridized to full-length cDNA of each gene labeled with $\mathrm{P}^{32}$ using the MEGA Labeling Kit (GE Healthcare, Piscataway NJ). DNA hybridizations were carried out at $65^{\circ} \mathrm{C}$ in $1 \mathrm{mM}$ ethylenediaminetetraacetic acid (EDTA), 7\% sodium dodecyl sulfate (SDS), 0.5 M sodium phosphate (pH7.2) for $16-18 \mathrm{~h}$. Filters were washed twice at $65^{\circ} \mathrm{C}$ in $1 \mathrm{mM}$ EDTA, 1\% SDS, $40 \mathrm{mM} \mathrm{Na}_{2} \mathrm{HPO}_{4}$ for 20 min, twice at $65^{\circ} \mathrm{C}$ in $1.5 \times$ sodium chloride/sodium citrate (SSC), $0.1 \%$ SDS for $20 \mathrm{~min}$ and twice at $65^{\circ} \mathrm{C}$ in $0.5 \times$ SSC, $0.1 \%$ SDS for $20 \mathrm{~min}$. Two or more BAC clones were identified for each gene and confirmed by PCR using plasmid DNA from individual colonies and gene specific primers. High purity plasmid DNA from individual BAC clones was then isolated using a NucleoBond BAC 100 kit (Macherey-Nagel Inc., Bethlehem, PA, USA) and both strands of DNA were sequenced using primers designed from the cDNAs and genomic DNA. DNA sequencing results were analyzed and assembled using Vector NTI software (Invitrogen, San Diego, CA), application Contig Assembly. The DNA sequence of each gene was then compared to the corresponding coding sequence by using the BLAST2 online tool (www.ncbi.nlm.nih.gov/ blast/bl2seq/wblast2.cgi) to obtain exon and intron locations for the gene organization analyses.

\section{Phylogenetic analysis}

Deduced protein sequences of all Arabidopsis IFR-like genes were retrieved from The Arabidopsis Information Resource (TAIR) database (http://www.arabidopsis.org/) by querying the TAIR protein database with the Desmodium LAR protein sequence (CAD79341) using the WU-BLAST2 (BLASTP) program. Protein sequences 
from other species were retrieved from the GenBank (http://www.ncbi.nlm.nih.gov/Genbank/). Accession numbers are indicated in the figure legend.

Multiple sequence alignment of proteins was performed by ClustalX algorithm [49] with default parameter settings (gap opening penalty: 10, gap extension penalty: 0.2 , delay divergent cutoff: $30 \%$, protein weight matrix: Gonnet series) and this alignment was used to construct the phylogenetic tree using the neighbor-joining method in the MEGA package [50]. Five hundred bootstrapped datasets were used to estimate the confidence of each tree clade.

\section{Determination of proanthocyanidins (PAs) and anthocyanins}

For quantitative analysis of anthocyanin levels in transgenic tobacco flowers, fresh petals (0.3-0.5 g fresh weight) from three flowers were immersed in $5 \mathrm{~mL}$ ethanol: $6 \mathrm{M} \mathrm{HCl}(1: 1)$ and incubated at $4^{\circ} \mathrm{C}$ overnight. The extract solution was transferred to a new tube and the petals were extracted for the second time using the same method. Absorbance of the pooled extract solution was then measured at $526 \mathrm{~nm}$ and the total anthocyanin levels were calculated using a standard molar absorbance curve prepared using cyanidin-3-glucoside (Sigma-Aldrich, MO, USA).

To extract soluble PAs from cacao and tobacco tissues, 0.3 to $0.5 \mathrm{~g}$ of frozen tissues were ground into a fine powder in liquid nitrogen and then extracted with $5 \mathrm{~mL}$ of extraction solution (70\% acetone: $29.5 \%$ water: $0.5 \%$ acetic acid) by vortexing for 5 seconds followed by water bath sonication for 15 min using a bench top ultrasonic cleaner (Model 2510, Bransonic, Danbury, CT, USA). To extract soluble PAs from Arabidopsis seeds and siliques, the same extraction solution and method were applied, except that 100 to $500 \mathrm{mg}$ dry seeds and 10 green siliques were used as grinding samples, and $500 \mu \mathrm{L}$ extraction solution were used. After sonication, samples were vortexed again and centrifuged at $2500 \mathrm{~g}$ for $10 \mathrm{~min}$. The supernatant was transferred to a new tube and the pellet was re-extracted twice as above. Pooled supernatants were extracted twice with hexane to remove fat and chlorophyll and then filtered through a $0.45 \mu \mathrm{m}$ polytetrafluoroethylene (PTFE) syringe filter (Millipore, Billerica, MA, USA). Depending on availability of plant samples, different numbers of biological replicates were performed for cacao, tobacco and Arabidopsis samples. For cacao, there are at least five biological replicates, for tobacco, there are seven or more biological replicates, and for Arabidopsis, there are three biological replicates.

To quantify soluble PA levels, extracts were then quantified by reaction with $p$-dimethylamino-cinnamaldehyde (DMACA), which specifically interacts with PA monomers and polymers to form blue pigments [51].
Briefly, $50 \mu \mathrm{L}$ aliquots of samples were mixed with $200 \mu \mathrm{L}$ of dimethylaminocinnamaldehyde (DMACA; Sigma-Aldrich, MO, USA) reagent (0.1\% DMACA, 90\% reagent-grade ethanol, $10 \% \mathrm{HCl}$ ) in 96-well microtiter plates. Absorption was measured at $640 \mathrm{~nm}$ at oneminute intervals for $20 \mathrm{~min}$ to get the highest readings. Triple technical replicates were performed to obtain mean values. The total PA levels were calculated using the standard molar absorbance curve prepared using procyanidin B2 (Indofine, NJ, USA).

For quantitative analysis of insoluble PAs from cacao tissues, the residues from soluble PA extractions were air dried in an exhaust hood for two days, weighed, and $5 \mathrm{~mL}$ butanol- $\mathrm{HCl}$ reagent (95\% butan-1-ol: $5 \%$ concentrated $\mathrm{HCl}$ ) was added and the mixture was sonicated for one hour followed by centrifugation at $2500 \mathrm{~g}$ for $10 \mathrm{~min}$. An aliquot of clear supernatant was diluted 40-fold in butanol- $\mathrm{HCl}$ reagent and absorbance was measured at $550 \mathrm{~nm}$ to determine the amount of background absorption. The samples were then boiled for 1 hour with vortexing every $20 \mathrm{~min}$, cooled to room temperature and centrifuged again at $2500 \mathrm{~g}$ for $10 \mathrm{~min}$. The supernatant from boiled sample was diluted 40-fold in butanol- $\mathrm{HCl}$ reagent and absorbance was measured at $550 \mathrm{~nm}$. The values were normalized by subtraction of the background absorbance and the PA levels were calculated as cyanidin equivalents using cyanidin-3-glucoside (SigmaAldrich, MO, USA) as standards.

To visualize the presence of PAs in Arabidopsis seeds, dry seeds were immersed for 2 days in the $0.1 \%$ DMACA reagent described above and then washed 3 times with $70 \%$ ethanol as described previously [35]. Catechin and epicatechin content was determined by reverse-phase HPLC using an Alliance separations module (Model 2695; Waters, Milford, MA, USA) equipped with a multi $\lambda$ fluorescence detector (Model 2475; Waters, Milford, MA, USA). Samples of soluble PA extracts $(10 \mu \mathrm{L})$ were separated on a $250 \mathrm{~mm} \times 4.6 \mathrm{~mm}$ Luna 5- $\mu \mathrm{m}$ Phenyl Hexyl column (Phenomenex, Torrance, CA, USA) and then assayed by fluorescence emission at $315 \mathrm{~nm}$ following excitation at $280 \mathrm{~nm}$. The HPLC separation utilized a binary mobile phase gradient mixture of $\mathrm{A}+\mathrm{B}$ where mobile phase $\mathrm{A}$ was $0.5 \%$ trifluoroacetic acid (TFA) (v/v with water) and mobile phase $B$ was $0.5 \%$ TFA (v/v with methanol). The gradient conditions were: $0 \mathrm{~min}, 16 \%$ mobile phase $\mathrm{B} ; 4 \mathrm{~min}, 16 \%$ mobile phase B; $14 \mathrm{~min}, 50 \%$ mobile phase B; $18 \mathrm{~min}$, $50 \%$ mobile phase $\mathrm{B} ; 22 \mathrm{~min}, 100 \%$ mobile phase $\mathrm{B} ; 26$ min, $100 \%$ mobile phase $\mathrm{B} ; 30 \mathrm{~min}, 16 \%$ mobile phase B. The column was maintained at $30^{\circ} \mathrm{C}$ and the flow rate was $1 \mathrm{~mL} / \mathrm{min}$. Catechin and epicatechin standards were purchased from Sigma-Aldrich (St. Louis, MO, USA). This work was performed at the Hershey Technical Center (Hershey, PA, USA). 


\section{Transformation of tobacco and arabidopsis}

The coding sequences of TcANS, TcANR and TcLAR were excised from the cloning vector (pGEM-T easy) (Promega, Madison, WI, USA) with NcoI and NotI restriction enzymes and cloned into the pE2113-EGFP [44] intermediate vector to replace the original EGFP coding sequence. As a result, the cacao coding sequences are located immediately downstream of the E12- $\Omega$, an enhanced expression promoter modified from CaMV35S [52], and upstream of the CaMV35S-terminator. The overexpression cassettes of TcANR and TcANS was excised out from pE2113-TcANR and pE2113-TcANS constructs respectively with HaeII restriction enzyme, blunt ended with T4 polymerase and then introduced into the pCAMBIA-1300 binary vector (CAMBIA, Canberra, Australia) linearized with SmaI restriction enzyme; the over-expression cassettes of TcLAR was excised out from pE2113-TcLAR construct with HindIII and PvuII restrictin enzyme and ligated into pCAMBIA-1300 binary vector linearized with HindIII and SmaI restriction enzyme. All binary transformation constructs were introduced into Agrobacterium tumefaciens strain AGL1 [53] by electroporation as described previously [54].

Tobacco leaf disc transformation was performed as previously described [55] and transgenic shoots were regenerated on MSs (MS shooting) media supplemented with $25 \mathrm{mg} / \mathrm{L}$ hygromycin. Only one shoot was selected from each explant to ensure independent transformants. After rooting for 2 weeks in MSr (MS rooting) media supplemented with $25 \mathrm{mg} / \mathrm{L}$ hygromycin, hygromycinresistant plantlets were transferred to soil and grown in a greenhouse as described above.

Arabidopsis transformation was carried out using the floral dip method [56], and T1 transgenic plants were selected on MS media supplemented with $2 \%$ sucrose, $0.65 \%$ agar and $25 \mathrm{mg} / \mathrm{L}$ hygromycin. Hygromycinresistant T1 seedlings were transferred to soil 7 days after germination and grown in a growth chamber as described in above.

\section{Expression analysis of TCANS, TCANR and TCLAR}

Total RNA from leaves, flowers, whole pods, pod exocarp and ovules of Theobroma cacao (Scavina 6 and Amelonado) was isolated as described above. Total RNA from young leaves of transgenic and wild-type tobacco plants as well as Arabidopsis plants was isolated using the RNeasy Plant mini kit (Qiagen, Valencia, CA, USA). cDNA was synthesized from $1 \mu \mathrm{g}$ of total RNA in a total volume of $20 \mu \mathrm{L}$ using M-MuLV Reverse Transcriptase (NEB, Ipswich, MA, USA) according to the supplier's protocols, and $2 \mu \mathrm{L}$ were used in the subsequent reverse transcription-PCR (RT-PCR) reactions. The primers for RT-PCR were designed to amplify across at least one intron giving products of approximately 500 bp from cDNA and $700 \mathrm{bp}$ to $1500 \mathrm{bp}$ from genomic DNA. These primer sets were used to check all cDNAs for genomic DNA contamination. The primers used for TcANS were TcANSRT_F (5'-ACCTTGTTAACCATG GGATCTCGG-3') and TcANSRT_R (5'-GACGGTGTCACCAATGTGCATGAT-3'); the primers used for TcANR were TcANR_F (5' - TGCTTGAGAAGGGCT ACGCTGTTA-3') and TcANR_R (5'-AAAGATGTGGCAAGGCCAATGCTG-3'); the primers used for TcLAR were TcLAR_F (5' -AATTCCATTGCAGCTTGGCCCTAC-3') and TcLAR_R (5'-GGCTTGCTCACTGCTT TGGCATTA-3'). TcActin was used as an internal standard for cacao gene expression using primer set Tc46RT_F (5'-AGCTGAGAGATTCCGTTGTCCAGA$3^{\prime}$ ) and Tc46RT_R (5' -CCCACATCAACCAGACTTT GAGTTC-3'). AtUbi and NtrRNA was chosen as constitutive expression controls for Arabidopsis (ubiquitin) and tobacco (rRNA) respectively with primer pairs AtUbi_F (5'-ACCGGCAAGACCATCACTCT-3') and AtUbi_R (5' -AGGCCTCAACTGGTTGCTGT-3') [57], and NtrRNA_F (5' -AGGAATTGACGGAAGGGCA-3') and NtrRNA_R (5'-GTGCGGCCCAGAACATCTAA G-3') [58].

The number of PCR cycles was optimized between 20 and 32 to select a cycle number such that amplification was in the linear range; 28 cycles were chosen for all the RT-PCR reactions. The PCR reaction was carried out in a total volume of $20 \mu \mathrm{L}$ at $94^{\circ} \mathrm{C}$ for $5 \mathrm{~min}$; 28 cycles of $94^{\circ} \mathrm{C}$ for $30 \mathrm{sec}, 55^{\circ} \mathrm{C}$ for $30 \mathrm{sec}$, and $72^{\circ} \mathrm{C}$ for $45 \mathrm{sec}$; followed by a final extension at $72^{\circ} \mathrm{C}$ for $5 \mathrm{~min}$. The PCR products were visualized on $1 \%$ agarose gels stained with ethidium bromide $(\mathrm{EtBr})$ and documented using Molecular Imager Gel Doc XR + System equipped with a 16-bit CCD camera (Bio-Rad Laboratories, Hercules, CA) and bands were quantified using Quantity One 1-D Analysis Software (Bio-Rad Laboratories, Hercules, CA).

\section{Assay of LAR activities}

The open reading frame (ORF) of the TcLAR gene was PCR amplified from pGEMT-TcLAR using Advantage 2 polymerase mix (Clontech, Mountain View, CA) and the following primers: TcLARCDF1 (5'-GAGCTCatggatatgaaatcaacaaacatg- $3^{\prime}$; the $S a c \mathrm{I}$ site is in italics and the start codon is bold) and TcLARCDR2 (5'-24 CTCGAGtgtgcatatcgcagtg-3'; the XhoI site is in italics and the stop codon was removed to incorporate the $C$ terminal His-tag sequence of the expression vector at the 3' end of the ORF of TcLAR). It was then subcloned into the $S a c$ I and XhoI sites of the pET-21a expression vector (Novagen, Gibbstown, NJ, USA). After confirmation by sequencing, the resulting vector pET21a-TcLAR was transformed into Escherichia coli strain Rosetta (DE3) (Novagen, Gibbstown, NJ, USA). For protein 
expression, a single bacterial colony was inoculated into Luria-Bertani medium (10 g/L tryptone, $5 \mathrm{~g} / \mathrm{L}$ yeast extract, $10 \mathrm{~g} / \mathrm{L} \mathrm{NaCl}$ ) containing $100 \mu \mathrm{g} / \mathrm{mL}$ ampicillin and grown at $37^{\circ} \mathrm{C}$ overnight. An overnight culture was then diluted into terrific broth (TB) medium (12 g/L Tryptone, $24 \mathrm{~g} / \mathrm{L}$ Yeast Extract, $0.4 \%$ glycerol, $2.31 \mathrm{~g} / \mathrm{L}$ $\mathrm{KH} 2 \mathrm{PO} 4,12.54 \mu \mathrm{g} / \mathrm{mLg} / \mathrm{L}$ K2HPO4) containing $100 \mathrm{ampi}-$ cillin and grown at $37^{\circ} \mathrm{C}$ until the OD600 reached 0.6-0.8, at which time IPTG (isopropyl $\beta$-D-1-thiogalactopyranoside), was added to a final concentration of $1 \mathrm{mM}$ to induce protein expression. Recombinant TcLAR protein with a 6. His tag at the $\mathrm{C}$ terminus was purified using a MagneHis kit (Promega, Madison, WI, USA) and the protein concentration measured by the Bradford method [59]. This work was performed at the Samuel Roberts Nobel Foundation (Ardmore, Oklahoma, USA).

${ }^{3} \mathrm{H}-3,4$-cis-leucocyanidin was synthesized as described by [60]. Assay of recombinant TcLAR protein with ${ }^{3} \mathrm{H}-3$, 4-cis-leucocyanidin was carried out in a final volume of $100 \mu \mathrm{L}$ containing $10 \%(\mathrm{w} / \mathrm{v})$ glycerol, $100 \mathrm{mM}$ potassium phosphate $(\mathrm{pH} 7.0), 4 \mathrm{mM}$ dithiothreitol (DTT), $0.5 \mathrm{mM}$ NADPH, $0.4 \mathrm{mM}{ }^{3} \mathrm{H}$-leucocyanidinugofpurifieand recombinant30 TcLAR protein. The reaction was initiated by the addition of enzyme and incubated at $30^{\circ} \mathrm{C}$ for $1 \mathrm{~h}$. The assay was terminated by the addition of $20 \mu \mathrm{L}$ of methanol followed by centrifugation. Products were analyzed by HPLC, with absorbance monitoring at $280 \mathrm{~nm}$. Products eluting at retention times between 13 to $31 \mathrm{~min}$ were collected (1 min/tube) and the fractions containing labeled products were identified by liquid scintillation counting. Boiled pure protein was used as a control.

Reverse-phase HPLC analysis of enzymatic products was performed using an Agilent HP1100 HPLC (Agilent Technologies, Inc., Santa Clara, CA, USA) with the following gradient using solvents $\mathrm{A}$ ( $1 \%$ phosphoric acid) and $\mathrm{B}$ (acetonitrile) at a $1 \mathrm{~mL} / \mathrm{min}$ flow rate: 0 to $5 \mathrm{~min}$, $6 \% \mathrm{~B} ; 5$ to $10 \mathrm{~min}, 6 \%$ to $10 \% \mathrm{~B} ; 10$ to $20 \mathrm{~min}, 10 \%$ to $11 \%$ B; 20 to $25 \mathrm{~min}, 11 \%$ to $12.5 \% \mathrm{~B} ; 25$ to $45 \mathrm{~min}, 12.5 \%$ to $37 \% \mathrm{~B} ; 45$ to $48 \mathrm{~min}, 37 \%$ to $100 \% \mathrm{~B}$; 48 to $58 \mathrm{~min}, 100 \%$, 58 to $60 \mathrm{~min} 100 \%$ to $6 \% \mathrm{~B}$. Absorbance data were collected at $280 \mathrm{~nm}$. Identifications were based on comparison of chromatographic behavior and UV spectra with authentic standards. This work was performed at Samuel Roberts Nobel Foundation (Ardmore, Oklahoma, USA).

Sequence data from this article can be found in the GenBank/EMBL data libraries under accession numbers GU324347 (TcANR), GU324349 (TcANS) and GU324351 (TcLAR).

\section{Additional file}

Additional file 1: Figure S1. Multiple sequence alignment of the $L A R$, ANS and ANR proteins as well as related IFR and DFR proteins of the RED superfamily. Figure S2. Complementation of the PA and anthocyanin deficient ans (Idox) mutant phenotype by constitutively expressing TCANS. Figure S3. HPLC analysis of extracts from transgenic Arabidopsis and tobacco key samples with and without standard spikes to validate the peak of catechin and epicatechin. Figure S4. Complementation of the PA deficient Arabidopsis Idox mutant phenotype by constitutive expression of TCLAR.

\section{Competing interests}

The authors declare that they have no competing interests.

\section{Authors' contributions}

$Y L$ performed most of the experiments, ie, sequence analysis, gene cloning, gene expression studies, transgenic tobacco and Arabidopsis generation, phenotypic analysis of transgenic lines, and drafted the manuscript. ZS participated in gene expression analysis, transgenic Arabidopsis lines generation and analysis. SNM participated in the design of the study, directed the vector construction and tobacco transgenic lines generation, and participated in drafting of the manuscript. MJP developed the catechin and epicatechin HPLC quantification assays and directed PA quantification analysis. MJG conceived the study, drafted the manuscript and gave advice on experimental design, data analysis and execution. All authors read and approved the final manuscript.

\section{Acknowledgments}

We thank Yongzhe Pang and Richard A. Dixon from Samuel Roberts Noble Foundation for valuable contribution on the TCLAR protein purification and enzyme assay, Dennis Arocena for assistance with PA and anthocyanin extraction, Dr. David Stuart and the scientists and staff from Hershey Center for Health and Nutrition for discussions and support through providing access to Hershey Technical Center HPLC facility, Ann Young and Sharon Pishak for assistance with green house maintenance, tissue culture and cacao sample collection, Kabing Xie and Qin Wang for valuable technical suggestions on TCLAR recombinant protein expression. This work is supported in part by the Hershey Foods Corporation, The Pennsylvania State University, The Huck Institutes of Life Sciences and American Research Institute Penn State Endowed Program in the Molecular Biology of Cacao.

\section{Author details}

${ }^{1}$ Huck Institute of Life Sciences, The Pennsylvania State University, University Park, PA 16802, USA. ²Department of Plant Science, The Pennsylvania State University, 422 Life Sciences Building, University Park, PA 16802, USA.

${ }^{3}$ Hershey Center for Health and Nutrition, The Hershey Company, 1025 Reese Ave, Hershey, PA 17033, USA. ${ }^{4}$ Present address: Cellular \& Molecular Pharmacology, Mission Bay Campus, Genentech Hall, University of California, San Francisco, N582/Box 2280, 600 16th Street, San Francisco, CA 94158, USA.

Received: 22 July 2013 Accepted: 29 November 2013

Published: 5 December 2013

\section{References}

1. Dixon RA, Xie DY, Sharma SB: Proanthocyanidins - a final frontier in flavonoid research? New Phytol 2005, 165:9-28.

2. Martin C, Prescott A, Mackay S, Bartlett J, Vrijlandt E: Control of anthocyanin biosynthesis in flowers of Antirrhinum majus. Plant J 1991, 1:37-49.

3. Saslowsky D, Winkel-Shirley B: Localization of flavonoid enzymes in Arabidopsis roots. Plant $J$ 2001, 27:37-48.

4. Winkel-Shirley B: Biosynthesis of flavonoids and effects of stress. Curr Opin Plant Biol 2002, 5:218-223.

5. Feucht W, Treutter D: The Role of Flavan-3-ols and Proanthocyanidins in Plant Defense. In Principles and Practices in Plant Ecology: Allelochemical Interactions. Edited by Inderjit, Dakshi KMM, Foy CL. New York: CRC Press; 1999:307-338.

6. Pourcel L, Routaboul JM, Cheynier V, Lepiniec L, Debeaujon I: Flavonoid oxidation in plants: from biochemical properties to physiological functions. Trends Plant Sci 2007, 12:29-36.

7. Pourcel L, Routaboul JM, Kerhoas L, Caboche M, Lepiniec L, Debeaujon I: TRANSPARENT TESTA10 encodes a laccase-like enzyme involved in oxidative polymerization of flavonoids in Arabidopsis seed coat. Plant Cell 2005, 17:2966-2980. 
8. Scalbert A: Antimicrobial properties of tannins. Phytochemistry 1991, 30:3875-3883.

9. Aron PM, Kennedy JA: Flavan-3-ols: nature, occurrence and biological activity. Mol Nutr Food Res 2008, 52:79-104.

10. Norman KH, Naomi DLF, Marjorie LM: Flavanols, the Kuna, cocoa consumption, and nitric oxide. J Am Soc Hypertens 2009, 3:105-112.

11. Ramassamy C: Emerging role of polyphenolic compounds in the treatment of neurodegenerative diseases: a review of their intracellular targets. Eur J Pharmacol 2006, 545:51-64.

12. Lepiniec $L$, et al: Genetics and biochemistry of seed flavonoids. Annu Rev Plant Biol 2006, 57:405-430.

13. Winkel-Shirley B: It takes a garden. How work on diverse plant species has contributed to an understanding of flavonoid metabolism. Plant Physiol 2001, 127:1399-1404.

14. Tanner GJ, Francki KT, Abrahams S, Watson JM, Larkin PJ, Ashton AR: Proanthocyanidin biosynthesis in plants. Purification of legume leucoanthocyanidin reductase and molecular cloning of its CDNA. J Biol Chem 2003, 278:31647-31656.

15. Abrahams S, Lee E, Walker AR, Tanner GJ, Larkin PJ, Ashton AR: The Arabidopsis TDS4 gene encodes leucoanthocyanidin dioxygenase (LDOX) and is essential for proanthocyanidin synthesis and vacuole development. Plant J 2003, 35:624-636

16. Saito K, Kobayashi M, Gong Z, Tanaka Y, Yamazaki M: Direct evidence for anthocyanidin synthase as a 2-oxoglutarate-dependent oxygenase: molecular cloning and functional expression of cDNA from a red form of Perilla frutescens. Plant J 1999, 17:181-189.

17. Xie DY, Sharma SB, Paiva NL, Ferreira D, Dixon RA: Role of anthocyanidin reductase, encoded by BANYULS in plant flavonoid biosynthesis. Science 2003, 299:396-399.

18. Schijlen EG, Ric de Vos CH, Van Tunen AJ, Bovy AG: Modification of flavonoid biosynthesis in crop plants. Phytochemistry 2004, 65:2631-2648

19. Pang Y, Peel GJ, Sharma SB, Tang Y, Dixon RA: A transcript profiling approach reveals an epicatechin-specific glucosyltransferase expressed in the seed coat of Medicago truncatula. Proc Natl Acad Sci 2008, 105:14210-14215.

20. Zhao J, Dixon RA: MATE transporters facilitate vacuolar uptake of epicatechin 3'-O-glucoside for proanthocyanidin biosynthesis in Medicago truncatula and Arabidopsis. Plant Cell 2009, 21:2323-2340.

21. Zhao J, Pang $Y$, Dixon RA: The mysteries of proanthocyanidin transport and polymerization. Plant Physiol 2010, 153:437-443.

22. Devic $M$, et al: The BANYULS gene encodes a DFR-like protein and is a marker of early seed coat development. Plant J 1999, 19:387-398.

23. Pelletier MK, Murrell JR, Shirley BW: Characterization of flavonol synthase and leucoanthocyanidin dioxygenase genes in Arabidopsis. Further evidence for differential regulation of "early" and "late" genes. Plant Physiol 1997, 113:1437-1445.

24. Pang Y, Peel GJ, Wright E, Wang Z, Dixon RA: Early steps in proanthocyanidin biosynthesis in the model legume Medicago truncatula. Plant Physiol 2007, 145:601-615.

25. Bogs J, Downey MO, Harvey JS, Ashton AR, Tanner GJ, Robinson SP: Proanthocyanidin synthesis and expression of genes encoding leucoanthocyanidin reductase and anthocyanidin reductase in developing grape berries and grapevine leaves. Plant Physiol 2005, 139:652-663.

26. Paolocci F, Robbins MP, Madeo L, Arcioni S, Martens S, Damiani F: Ectopic expression of a basic helix-loop-helix gene transactivates parallel pathways of proanthocyanidin biosynthesis. structure, expression analysis, and genetic control of leucoanthocyanidin 4-reductase and anthocyanidin reductase genes in Lotus corniculatus. Plant Physiol 2007, 143:504-516.

27. Foo LY, Newman R, Waghorn G, McNabb WC, Ulyatt MJ: Proanthocyanidins from lotus corniculatus. Phytochemistry 1996, 41:617-624.

28. Chaves FC, Gianfagna TJ: Cacao leaf procyanidins increase locally and systemically in response to infection by Moniliophthora perniciosa basidiospores. Physiol Mol Plant Pathol 2007, 70:174-179.

29. Niemenak N, Rohsius C, Elwers S, Ndoumou DO, Lieberei R: Comparative study of different cocoa (Theobroma cacao L.) clones in terms of their phenolics and anthocyanins contents. J Food Comp Anal 2006, 19:612-619.

30. Gu L, et al: Fractionation of polymeric procyanidins from lowbush blueberry and quantification of procyanidins in selected foods with an optimized normal-phase HPLC-MS fluorescent detection method. J Agric Food Chem 2002, 50:4852-4860.

31. Argout $X$, et al: Towards the understanding of the cocoa transcriptome: production and analysis of an exhaustive dataset of ESTs of Theobroma cacao $L$. Generated from various tissues and under various conditions. BMC Genomics 2008, 9:512.

32. Argout X: The genome of Theobroma cacao. Nat Genet 2011, 43:101.

33. Li YG, Tanner G, Larkin P: The DMACA-HCl protocol and the threshold proanthocyanidin content for bloat safety in forage legumes. J Sci Food Agric 1996, 70:89-101.

34. Treutter D: Significance of flavonoids in plant resistance and enhancement of their biosynthesis. Plant Biol 2005, 7:581-591.

35. Abrahams S, Tanner GJ, Larkin PJ, Ashton AR: Identification and biochemical characterization of mutants in the proanthocyanidin pathway in Arabidopsis. Plant Physiol 2002, 130:561-576.

36. Alemanno L, Berthouly M, MichauxFerriere N: A comparison between Theobroma cacao L. Zygotic embryogenesis and somatic embryogenesis from floral explants. In vitro cell. Dev Biol Plant 1997, 33:163-172.

37. Cheesman EE: Fertilization and embryogeny in Theobroma cacao, L. Ann Bot 1927, 41:107-126.

38. Wright DC, Park WD, Leopold NR, Hasegawa PM, Janick J: Accumulation of lipids, proteins, alkaloids and anthocyanins during embryo development in vivo of Theobroma cacao L. J Am Oil Chem Soc 1982, 59:475-479.

39. Winkel BS: Metabolic channeling in plants. Annu Rev Plant Biol 2004, 55:85-107.

40. Gargouri M, et al: Structure and epimerase activity of anthocyanidin reductase from Vitis vinifera. Acta Crystallogr D 2009, 65:989-1000.

41. Pang $Y Z$, et al: Functional characterization of proanthocyanidin pathway enzymes from Tea and their application for metabolic engineering. Plant Physiol 2013, 161:1103-1116.

42. Szankowski l, et al: Shift in polyphenol profile and sublethal phenotype caused by silencing of anthocyanidin synthase in apple (Malus sp.). Planta 2009, 229:681-692.

43. Loake GJ, Choudhary AD, Harrison MJ, Mavandad M, Lamb CJ, Dixon RA: Phenylpropanoid pathway intermediates regulate transient expression of a chalcone synthase gene promoter. Plant Cell 1991, 3:829-840.

44. Maximova S, Miller C, Antunez de Mayolo G, Pishak S, Young A, Guiltinan MJ: Stable transformation of Theobroma cacao $L$. and influence of matrix attachment regions on GFP expression. Plant Cell Rep 2003, 21:872-883

45. Bailey BA, et al: Developmental expression of stress response genes in Theobroma cacao leaves and their response to Nep1 treatment and a compatible infection by Phytophthora megakarya. Plant Physiol Biochem 2005, 43:611-622.

46. Murashige T, Skoog F: A revised medium for rapid growth and bio assays with tobacco tissue cultures. Physiol Plant 1962, 15:473-497.

47. Verica JA, Maximova SN, Strem MD, Carlson JE, Bailey BA, Guiltinan MJ: Isolation of ESTs from cacao (Theobroma cacao L.) leaves treated with inducers of the defense response. Plant Cell Rep 2004, 23:404-413.

48. Altschul SF, Gish W, Miller W, Myers EW, Lipman DJ: Basic local alignment search tool. J Mol Biol 1990, 215:403-410.

49. Thompson JD, Gibson TJ, Plewniak F, Jeanmougin F, Higgins DG: The CLUSTAL_X windows interface: flexible strategies for multiple sequence alignment aided by quality analysis tools. Nucleic Acids Res 1997 25:4876-4882.

50. Kumar S, Tamura K, Nei M: MEGA3: integrated software for molecular evolutionary genetics analysis and sequence alignment. Brief Bioinform 2004, 5:150-163.

51. Thies M, Fischer R: New color reaction for microchemical detection and quantitative determination of catechins. Mikrochim Acta 1971, 1:9-13.

52. Mitsuhara l, et al: Efficient promoter cassettes for enhanced expression of foreign genes in dicotyledonous and monocotyledonous plants. Plant Cell Physiol 1996, 37:49-59.

53. Lazo GR, Stein PA, Ludwig RA: A DNA transformation-competent Arabidopsis genomic library in Agrobacterium. Biotechnology (N Y) 1991 9:963-967.

54. Lin JJ: Optimization of the transformation efficiency of Agrobacterium tumefaciens cells using electroporation. Plant Sci 1994, 101:11-15.

55. Fisher DK, Guiltinan MJ: Rapid, efficient production of homozygous transgenic tobacco plants with agrobacterium tumefaciens: a seed-to-seed protocol. Plant Mol Biol Reptr 1995, 13:278-289. 
56. Clough SJ, Bent AF: Floral dip: a simplified method for Agrobacteriummediated transformation of Arabidopsis thaliana. Plant J 1998, 16:735-743.

57. Zhang W, Sun Y, Timofejeva L, Chen C, Grossniklaus U, Ma H: Regulation of Arabidopsis tapetum development and function by DYSFUNCTIONAL TAPETUM1 (DYT1) encoding a putative bHLH transcription factor. Development 2006, 133:3085-3095.

58. Levy M, Edelbaum O, Sela I: Tobacco mosaic virus regulates the expression of its own resistance gene N. Plant Physiol 2004, 135:2392-2397.

59. Bradford MM: A rapid and sensitive method for the quantitation of microgram quantities of protein utilizing the principle of protein-dye binding. Anal Biochem 1976, 72:248-254.

60. Tanner GJ, Kristiansen KN: Synthesis of 3,4-cis-[3H]leucocyanidin and enzymatic reduction to catechin. Anal Biochem 1993, 209:274-277.

\section{doi:10.1186/1471-2229-13-202}

Cite this article as: Liu et al.: Proanthocyanidin synthesis in Theobroma cacao: genes encoding anthocyanidin synthase, anthocyanidin reductase, and leucoanthocyanidin reductase. BMC Plant Biology 2013 13:202.

\section{Submit your next manuscript to BioMed Central and take full advantage of:}

- Convenient online submission

- Thorough peer review

- No space constraints or color figure charges

- Immediate publication on acceptance

- Inclusion in PubMed, CAS, Scopus and Google Scholar

- Research which is freely available for redistribution 